\title{
Bufferless Bidirectional Multi-Ring Networks with Sharing an Optical Burst Mode Transceiver for Any Route
}

\author{
Kyota HATTORI $^{\dagger a)}$, Masahiro NAKAGAWA ${ }^{\dagger}$, Toshiya MATSUDA ${ }^{\dagger}$, Masaru KATAYAMA ${ }^{\dagger}$, Members, $^{\circ}$ \\ and Katsutoshi KODA ${ }^{\dagger}$, Nonmember
}

\begin{abstract}
SUMMARY Improvement of conventional networks with an incremental approach is an important design method for the development of the future internet. For this approach, we are developing a future aggregation network based on passive optical network (PON) technology to achieve both cost-effectiveness and high reliability. In this paper, we propose a timeslot (TS) synchronization method for sharing a TS from an optical burst mode transceiver between any route of arbitrary fiber length by changing both the route of the TS transmission and the TS control timing on the optical burst mode transceiver. We show the effectiveness of the proposed method for exchanging TSs in bidirectional bufferless wavelength division multiplexing (WDM) and time division multiplexing (TDM) multi-ring networks under the condition of the occurrence of a link failure through prototype systems. Also, we evaluate the reduction of the required number of optical interfaces in a multi-ring network by applying the proposed method.

key words: time division multiplexing, timeslot synchronization, bidirectional bufferless WDM/TDM multi-ring networks
\end{abstract}

\section{Introduction}

With the growing popularity of the internet, expectations for new applications and services in various fields such as multi-media and transportation have increased. These have motivated the development of the future internet, which will achieve higher bandwidth, lower delay, higher reliability, and greater intelligence to accommodate future services. To create the future internet, many research programs such as FI-PPP [1] of the EU, FIA-NP [2], and US Ignite [3] of the U.S. have been founded and several drastically new network architectures for the future internet proposed. However, these programs haven't led to any fundamental changes in the basic underlying architecture because of the difficulty in maintaining the same operation for the current system and the increase in capital expenditure (CAPEX) needed to replace the entire existing infrastructure with new architecture. Therefore, for the time being, it is expected that the incremental approach for the current network will be effective. This is why we are focusing on architecture based on the current network system.

The current network, which is a three-layer structure, consists of core, aggregation, and access network. The basic function of the core network is to accommodate large amounts of traffic by setting the optical path between edges

Manuscript received July 15, 2016.

Manuscript revised December 19, 2016.

Manuscript publicized February 8, 2017.

${ }^{\dagger}$ The authors are with NTT Network Service Systems Laboratories, NTT Corporation, Musashino-shi, 180-8585 Japan.

a) E-mail: hattori.kyota@lab.ntt.co.jp

DOI: 10.1587/transinf.2016NTP0001 of core network. Currently, a 100 Gbps interface is commercially available for the bandwidth of a wavelength in the core network. Moreover, it is predicted that optical networks will be required to support Tbps class transmission in the near future [4]. In response to this, research and development are also progressing [5] according to these requirements. On the other hand, the requirements for the future internet will severely affect the access network and the aggregation network. Because this network equipment has to be implemented at lower CAPEX in terms of the number of accommodated users per unit. In the access network, fiber to the home (FTTH) based on the passive optical network (PON) [6] has grown rapidly. When FTTH deployment in the access network has been accelerating, the operators and vendors are looking ahead to meet the requirements of new applications. A promising solution is to develop a higher speed PON such as the 10 Gigabit Ethernet PON (10GEPON) [7]. Recently, the standardization of the next generation of EPON (NG-EPON) [8] as a future access network has started to be discussed. These approaches are broadbanding of the access network. Other studies have applied optical burst control techniques used in the PON to the aggregation network. As one of these studies, an access aggregation convergence network has proposed by the application of long reach PON to the aggregation network in terms of the reduction of the aggregation network's CAPEX [9]. However, the application of long reach PON has resulted in several issues, such as optical burst mode amplifications to support long reach with lower CAPEX [10] and a decrease in the network delay caused by dynamic bandwidth allocation (DBA) [11].

As a different method to achieve a cost-effective aggregation network, rather than a converged approach for the access network in terms of current network architecture, we have been studying an approach to incorporate the optical burst control techniques used in the PON, such as 10GEPON, to the aggregation network, specifically, the optical Layer-2 switch network (OL2SW-NW) [12]-[18]. OL2SWNW was designed based on the application of bufferless optical time division multiplexing (TDM) and DBA to achieve a large-scale cost-effective aggregation NW. OL2SW-NW is named after the possession of the function of the Layer-2 switch (L2SW). In general, the L2SW switches data frames from input ports to output ports based on the Layer-2 destination address. Therefore, the L2SW learns Layer-2 addresses automatically to build a forwarding table that can 
be used to selectively forward data frames. In the same way, the OL2SW-NW acts as a large-scale L2SW covering the whole aggregation network in which the internal bandwidth for each destination is variable. OL2SW-NW can multiplex the traffic without increase in network delay through the application of a high speed timeslot (TS) allocation (TSA) algorithm [15], which expands the PON's DBA, for the large scale of the aggregation network. One of the advantages of applying these access network technologies to the aggregation network is that it makes it possible to reduce the CAPEX. Because access network devices are mass-produced, they must have a low CAPEX. Moreover, the OL2SW-NW can eliminate the internally located L2SW by aggregating the packet buffers to the entity of the edge node. As a result, the entity located at the edge of the OL2SW-NW has packet buffers and converts variable length Ethernet frames from the external network into optical burst mode signals in the same way as the PON. On the other hand, the inside of the OL2SW-NW switches the TSs with no packet buffer and without classifying the packet header. To achieve this architecture, it is necessary to synchronize the TS control timing among all nodes in the OL2SW-NW as there is no packet buffer in the internal node (Here, we use the term "TS $S_{\text {Sync }}$ " for "synchronization of TS control timing." A definition of the status of $\mathrm{TS}_{\text {Sync }}$ is described in Sect. 2.2.). Moreover, the aggregation network requires high reliability because it must accommodate larger number of users than the access network. Therefore, in this paper, we propose a reliable $\mathrm{TS}_{\text {Sync }}$ for the OL2SW-NW to achieve future aggregation networks. "Reliable $\mathrm{TS}_{\text {Sync" }}$ means that it is possible to continue to achieve $\mathrm{TS}_{\text {Sync }}$ with no packet buffer in internal nodes of arbitrary length and multiple routes when a link failure occurs.

\subsection{Related Works}

In current aggregation networks, optical add/drop multiplexer (OADM) systems based on wavelength division multiplexing (WDM) technology [19] have been widely deployed. However, an OADM performs add/drop at each wavelength and allocates the bandwidth by setting the optical path to fit the maximum amount of traffic from edge to edge of the aggregation network. Therefore, bandwidth utilization is a problem when accommodating a smaller traffic volume in comparison with the bandwidth of a wavelength. To overcome this problem, OADM systems are used in combination with traffic grooming technology to perform efficient traffic multiplexing. Traffic grooming is executed on mainly two levels: on the optical transport network (OTN) [20] as layer 1 and at the Ethernet switch as layer 2. The OTN can multiplex the traffic for all data units, which are called "optical data units (ODUs)," at an ODU cross-connect (XC). The Ethernet switch uses the media access control (MAC) address to decide where to forward Ethernet frames. These make it possible to reduce the number of optical interfaces $\left(N_{I F}\right)$ connected to the ODU-XC or the Ethernet switch at the aggregation point.
However, to achieve traffic grooming by using these technologies, an inter-connection node that executes traffic grooming needs to drop the traffic in order to multiplex the traffic and retransmit the traffic to the next node. As a result, $N_{I F}$ at the inter-connection node increases. One method for multiplexing traffic at the optical layer is to use a packetoptical add/drop multiplexer (P-OADM) system [21], [22]. $\mathrm{P}-\mathrm{OADM}$ extracts the control information from a packet and transports the control information via a control channel so as not to have to demodulate all the wavelengths at each node. This control channel generates the control timing of TSs and allocates the bandwidth for each node. However, P-OADM only synchronizes the control timing of TSs at single ring networks using a control channel. Therefore, when P-OADM is applied to a multi-ring network, it requires packet buffers at intermediate nodes connecting each ring [21]. Addressing this problem, our proposed OL2SWNW does not require packet buffers at intermediate nodes connecting each ring by achieving $\mathrm{TS}_{\text {Sync }}$ among all nodes. This can eliminate not only the packet buffers and packet classifiers but also $N_{I F}$ in order to insert the packets in the TS to the packet buffers in the intermediate nodes. However, previous proposed network has only supported unidirectional multi-ring network [14], which are not restorable for link and node failures. Therefore, there remains a challenge for an increase in reliability.

In view of the above background, the contribution of this paper is the achievement of a reliable $\mathrm{TS}_{\text {Sync }}$ for the OL2SW-NW. Figure 1 shows the concept of the reliable OL2SW-NW. The concept of the proposed network is a buffer-less optical TDM network to increase reliability while avoiding a complicated operation. Specifically, the proposed network aims to keep exchanging TSs between any route of arbitrary fiber length in bufferless optical TDM multi-ring networks even if a link failure occurs. This can eliminate the need for the operation to adjust the length of fiber. To achieve this, the proposed method dynamically changes both the route of the TS transmission and the TS control timing due to link failure. With this mechanism, the proposed method can eliminate the need to adjust the length of the fiber between all nodes, which bothers network operators.

The remainder of this paper is organized as follows. The architecture of the proposed network is described in

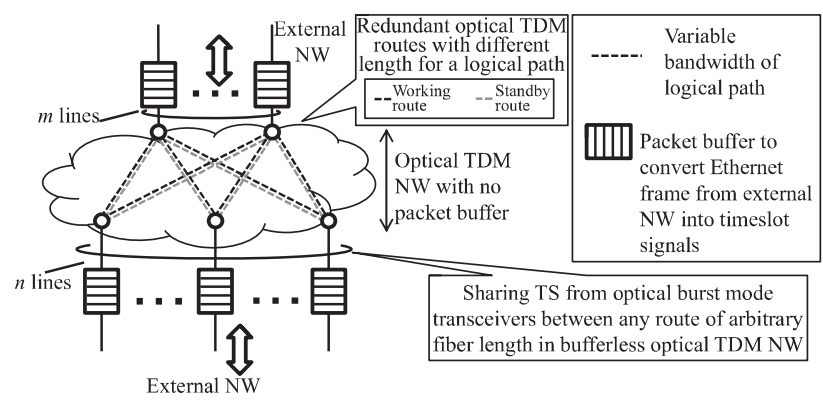

Fig. 1 Concept of reliable optical Layer-2 SW network. 
Sect. 2, and the proposed TS synchronization method, which is the key function for reliable OL2SW-NW, is described in Sect. 3. In Sect. 4, the accuracy and scalability of TS synchronization are described to evaluate the effective bandwidth for the proposed method. In Sect. 5, we explain the characteristic of $N_{I F}$ for the proposed method compared with the conventional method; P-OADM, which has to prepare packet buffers in the intermediate nodes connecting rings. The experimental and simulation results are described in Sect. 6. We conducted the experiments on the speed of changing TS control timings and the accuracy of TS synchronization to show the feasibility of the proposed method and the effective bandwidth using prototype systems. Then we showed comparisons of the simulation results of required $N_{I F}$ in the proposed method with those of P-OADM to evaluate the CAPEX. Finally, we concluded this paper in Sect. 7.

\section{Architecture of Reliable OL2SW-NW}

\subsection{Overview}

Figure 2 shows the architecture of a reliable OL2SWNW that can cost-effectively accommodate traffic in future aggregation networks based on a 10G-EPON system [7]. The OL2SW-NW is a bidirectional bufferless WDM/TDM multi-ring network located between access and core networks. It can characteristically eliminate the L2SW located in the internal network, which is required for conventional aggregation networks, by aggregating the packet buffer to the entity of the edge node in the OL2SW-NW. This entity is called an "Ether-burst converter (EBC) [13]," and it converts variable-length $10 \mathrm{G}$ Ethernet (10GE) frames to TSs with a fixed length and transmits them according to the allocated TSs at a constant TDM frame (the length of TDM frame: $\left.L_{F R}\right)$. Each node deploys a WDM/TDM SW [12], which enables TS add/drop for any channel and any TS. To allocate the bandwidth for logical paths between EBCs, the OL2SWNW has a TS scheduler that collects the traffic information on all logical paths for TSA calculated by a high speed TSA algorithm [13]. Then, the TS scheduler periodically allocates bandwidth at a constant cycle, $T$, which is an integral multiple of $L_{F R}$ for achieving DBA for large-scale aggregation networks [18]. The TS transmission at the EBC and the WDM/TDM SW are controlled by a TS controller (TSC), which is implemented at each node. TSCs at all nodes have to be $\mathrm{TS}_{\text {Sync }}$ because there is no packet buffer in the internal entity of the OL2SW-NW. Therefore, the OL2SW-NW has to achieve $\mathrm{TS}_{\text {Sync }}$ for the whole network. To achieve this, it has a TS synchronizer, which is set to aggregately execute $\mathrm{TS}_{\text {Sync }}$ for all TSCs. The TS synchronizer controls the TS control timing for all entities of the OL2SW-NW of arbitrary fiber lengths and arbitrary routes. This means that the TS synchronizer sets the start timing of the TDM frame and $T$ to all TSCs in consideration of the length of the routes of TS transmission. The method of communication between the TS synchronizer and all TSCs is using control channel $(\lambda c)$ to ensure reachability between each node connected by

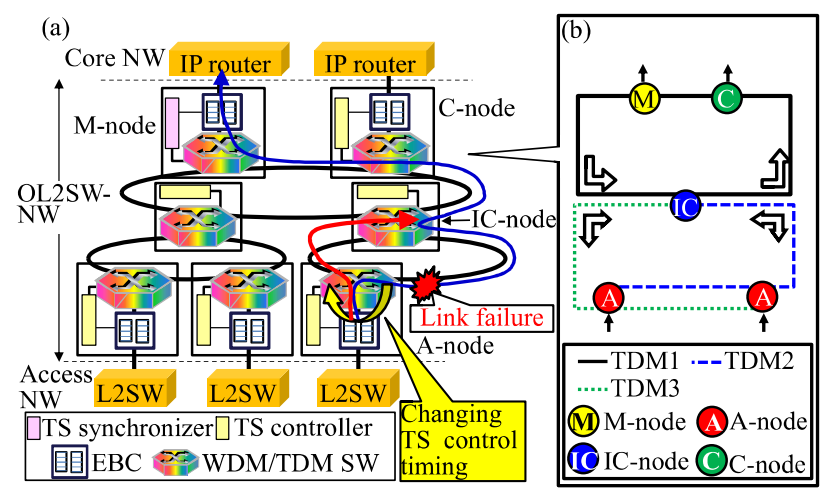

Fig. 2 Reliable optical Layer-2 SW network; (a) network architecture and (b) example of TS transmission from A-nodes to an upper ring.

an active WDM/TDM SW. We use two kinds of $\lambda \mathrm{c}: \lambda \mathrm{c} 1$ to send the control packets from the TS synchronizer to the TSC and $\lambda \mathrm{c} 2$ to send the control packets from the TSC to the TS synchronizer. $\lambda \mathrm{c} 1$ carries the control packets to synchronize the TS control timings and to set the information of allocated TSs. By contrast, $\lambda \mathrm{c} 2$ carries the control packets to measure the delay between the TS synchronizer and TSCs, the traffic information from external nodes connected to OL2SW-NW. Each $\lambda \mathrm{c}$ defines TSs, named control TS. Each TSC is allocated to a dedicated control TS in advance. All control information between the TS synchronizer and TSCs is carried using the control TSs

As described in Sect.1, the OL2SW-NW acts as a large-scale L2SW. To achieve this, the TS scheduler undertakes MAC learning [18], which is a necessary mechanism to perform as a L2SW. This mechanism is achieved as follows. The TSC checks the source MAC address from the arriving packets from external nodes connected to OL2SWNW and compares it to its MAC table. If it is a new address, the TSC notifies the TS scheduler of this address. The TS scheduler creates a new MAC table based on both the new MAC address and the TSC which the new external node is connected to. Then, the TS scheduler sets this MAC table to each TSC.

Here, we label the nodes that constitute the OL2SWNW; that is, the node connected to L2SWs in the access network is called an "A-node," the node connected to IP routers in the core network is called a "C-node," and the intermediate node connecting rings is called an "IC-node." Also, we call the node that deploys the TS scheduler and the TS synchronizer a "master node (M-node)."

\subsection{Definition of $\mathrm{TS}_{\text {Sync }}$}

An OL2SW-NW is required for $\mathrm{TS}_{\text {Sync }}$ in all nodes because of the elimination of packet buffers located in the internal network. Firstly, to explain the status of $\mathrm{TS}_{\text {Sync }}$, we define the TS control timing that each node has to prepare. Each node has the TS control timing in order to both control the arrival TS from the other nodes and send the TS from its own node. Figure 3 shows the definition of TS control 
timing, which is working at a node on a TDM frame consisting of four TSs. The length of TS $\left(: L_{T S}\right)$ is at a constant. TS control timing means the timing of each TS section which the node manages. Each node can produce the TS control timing according to its own clock frequency $(F q)$ by setting the start timing for the TDM frame, $L_{T S}$ and $L_{F R}$. In the OL2SW-NW, there is no packet buffer in the internal network. This means the TS gets to the receiver in the propagation delay between the sender and the receiver after the sender sends this TS. Therefore, to exchange TS in the OL2SW-NW, the TS control timing at the receiver must be delayed by the propagation delay in comparison to that of the sender. We define this status as $\mathrm{TS}_{\text {Sync }}$ (synchronization of the TS control timing). Figure 4 shows the definition of $\mathrm{TS}_{\text {Sync }}$ between the sender and receiver to exchange TSs. The status of $\mathrm{TS}_{\text {Sync }}$ means that the TS control timing at the receiver is delayed by the propagation delay in comparison to that of the sender. Moreover, because of the application of DBA in the OL2SW-NW, it is required to synchronize the start timing of $T$.

\subsection{Problem with $\mathrm{TS}_{\text {Sync }}$ in Reliable OL2SW-NW}

A reliable OL2SW-NW has to change to a standby route that does not go through the failure point to resume communication, if a link failure occurs over a working route that has already done $\mathrm{TS}_{\text {Sync }}$ between all nodes. However, if there is a difference in the length of the route of TS transmission between the working and standby routes, the start timing of the TDM frame shifts according to the difference in the length of these routes in the case that each node keeps working at the TS control timing for the working route. As a result, the arrival timing of TS at each node via the standby route deviates from that of the controlling TS at each node. Therefore, each node cannot forward the arriving TS.

A method for achieving $\mathrm{TS}_{\text {Sync }}$, which sets all fiber delays to an integral multiple of the TS between each node, was proposed [23]. To make this method a possibility, it is necessary to measure and adjust the delay between all nodes. However, it is complicated to adjust the length of a fiber, so this method is impractical for commercial
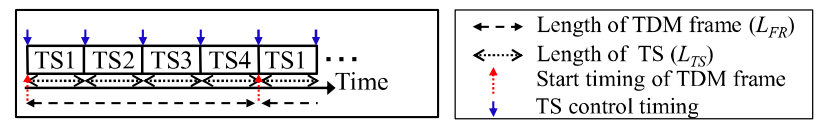

Fig. 3 Definition of TS control timing.

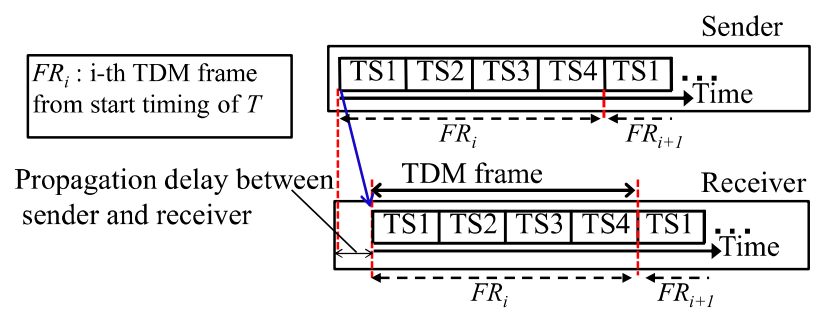

Fig. 4 Definition of $\mathrm{TS}_{\text {Sync }}$. networks. As a practical method, reference [14] proposed a method for supporting arbitrary fiber lengths between all nodes. This method executes $\mathrm{TS}_{\text {Sync }}$ between all nodes of arbitrary fiber lengths by separating the different TS control timings for the add and the drop at each node. This means that this method accommodates the different TS control timings for downstream and upstream traffic. However, when applying this method to bidirectional bufferless WDM/TDM multi-ring networks to enhance the reliability of an aggregation network, the required number of optical burst mode transceivers increases depending on the number of supportive routes. This means that [14] has to statically allocate both the transceivers for each TS control timing to all routes. Hence, the problem of [14] is reducing $N_{I F}$ without dependence on the number of routes.

\section{Proposed TS Sync $_{\text {Method }}$}

To achieve TS exchange while sharing an optical burst mode transceiver between arbitrary routes in bufferless WDM/TDM networks, the proposed method dynamically changes the route of the TS transmission and the TS control timing of both the WDM/TDM SW and the EBC for a link failure. This allows a TS from an EBC to be shared sequentially between any route of arbitrary fiber length working on different TS control timings. These TS control timings are calculated on the basis of the measured delay between each node. This method is an extension of our previous method [14] for application to bidirectional bufferless multi-ring networks. This method can reduce the number of transceivers compared with [14].

\section{1 $\mathrm{TS}_{\text {Sync }}$ in Arbitrary Fiber Lengths and Arbitrary Routes}

The basic idea of the proposed method for achieving $\mathrm{TS}_{\text {Sync }}$ between all nodes in arbitrary fiber lengths and arbitrary routes is based on separating the different TS control timings for the add and the drop at both the $\mathrm{C}$-node and A-node. Figure 5 shows the basic idea of the proposed method. In this

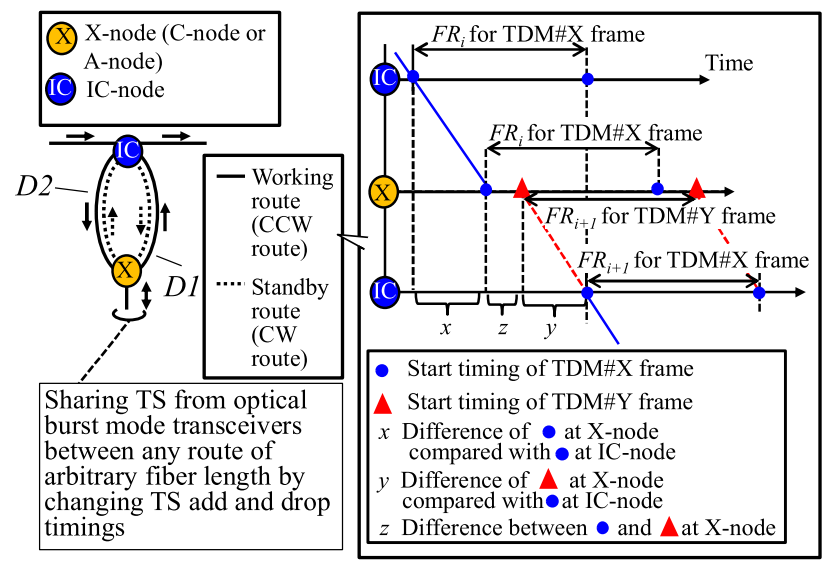

Fig. 5 Abstraction of proposed $\mathrm{TS}_{\text {Sync }}$ method. 


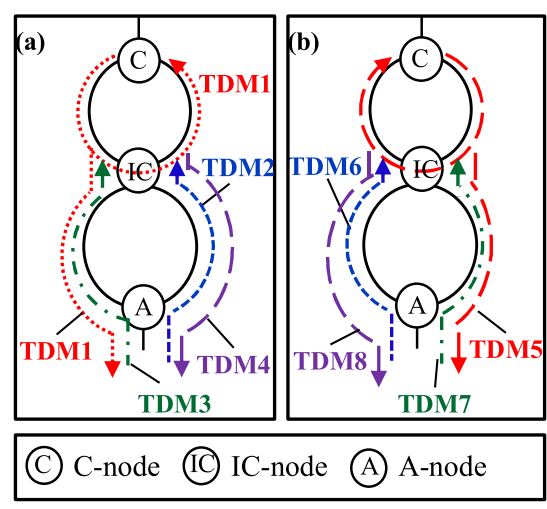

\begin{tabular}{|l|l|l|}
\hline $\begin{array}{l}\text { TS } \\
\text { control } \\
\text { timing }\end{array}$ & Utilization at C-node and IC-node & Utilization at A-node \\
\hline TDM1 & $\begin{array}{l}\text { To add and drop TS via CCW } \\
\text { route at the upper ring }\end{array}$ & $\begin{array}{l}\text { To drop TS via CCW route of the upper ring and CCW } \\
\text { route of the lower ring }\end{array}$ \\
\hline TDM2 & - & $\begin{array}{l}\text { To add TS via CCW route of the upper ring and CCW route } \\
\text { of the lower ring }\end{array}$ \\
\hline TDM3 & - & $\begin{array}{l}\text { To add TS via CCW route of the upper ring and CW route } \\
\text { of the lower ring }\end{array}$ \\
\hline TDM4 & - & $\begin{array}{l}\text { To drop TS via CCW route of the upper ring and CW route } \\
\text { of the lower ring }\end{array}$ \\
\hline TDM5 & $\begin{array}{l}\text { To add and drop TS via CW route } \\
\text { at the upper ring }\end{array}$ & $\begin{array}{l}\text { To drop TS via CW route of the upper ring and CW route } \\
\text { of the lower ring }\end{array}$ \\
\hline TDM6 & - & $\begin{array}{l}\text { To add TS via CW route of the upper ring and CW route of } \\
\text { the lower ring }\end{array}$ \\
\hline TDM7 & - & $\begin{array}{l}\text { To add TS via CW route of the upper ring and CCW route } \\
\text { of the lower ring }\end{array}$ \\
\hline TDM8 & - & $\begin{array}{l}\text { To drop TS via CW route of the upper ring and CCW route } \\
\text { of the lower ring }\end{array}$ \\
\hline
\end{tabular}

Fig. 6 Required TS control timings on bufferless two-stage rings network (a) for $\mathrm{CCW}$ route on the upper ring and (b) for $\mathrm{CW}$ route on the upper ring.

scenario, the OL2SW-NW has two kinds of fibers in both the upper ring and the lower ring. Each fiber carries traffic in one direction: a clockwise route (CW; standby route) or counter-clockwise route (CCW; working route). Each route has both a Data plane (D-plane) and Control plane (Cplane). The $\mathrm{CCW}$ route for the D-plane is used for normal operation, and the $\mathrm{CW}$ route for the D-plane is for failure recovery in the case of single link failure. On the other hand, both the $\mathrm{CCW}$ route for the $\mathrm{C}$-plane and the $\mathrm{CCW}$ route for the C-plane are used for normal operation. An up-/downstream path pair is carried on at the same direction. Here, as an example of $\mathrm{TS}_{\mathrm{Syn}}$, the $\mathrm{X}$-node belonging to the lower node executes $\mathrm{TS}_{\text {Sync }}$ for the IC-node. This means that the TS control timings at the $\mathrm{X}$-node have to be synchronized to that of the IC-node via both the CCW route and the $\mathrm{CW}$ route of arbitrary lengths. The TS control timings are used according to the route of TS transmission. These TS control timings are set to each node in advance. The key point of the proposed method is how to set these TS control timings and how to change the TS control timing for a protection route when a link failure occurs. Figure 5 shows an example of the TS control timings which X-node has; namely TDM\#X and TDM\#Y. To synchronize the TS control timings among all the nodes, the TS synchronizer set a baseline TS control timing, which is TDM\#X, to all nodes. Then, other TS control timings; TDM\#Y, are set based on TDM\#X. In the case of Fig. 5, TDM\#X is used to drop the TS via the CCW route and TDM\#Y is used to add the TS via the CCW route at the X-node (although the simplified explanation in Fig. 5 could not convey it, the X-node has as many TS control timings as the routes of TS transmission which are described in Sect. 3.2). Following, we show the relationship between the TS drop timing at the $\mathrm{X}$-node via the $\mathrm{CCW}$ route and the TS add timing at the $\mathrm{X}$-node via the $\mathrm{CCW}$ route. To synchronize the TS drop timing at the $\mathrm{X}$-node to the TS control timing at the IC-node, the TS drop timing in the X-node is set to be delayed by the propagation delay from the IC-node to the X-node in comparison with that of the IC-node. This delayed is expressed as $x$. To synchronize the TS add timing at the X-node to the TS control timing at the IC-node, the TS add timing at the $\mathrm{X}$-node is set to be earlier by the propagation delay from the $\mathrm{X}$-node to the IC-node in comparison to that of the IC-node. This is expressed as $y$. Also, we define the difference between $x$ and $y$ as $z$. If both the setting of TS drop timing as a baseline TS control timing and the calculation of $z$ are achieved based on the propagation delay between each node, it is possible to acquire the TS control timings for each route at the $\mathrm{X}$-node. For example, in the case that the propagation delay of the left-hand and right-hand routes are $D 2$ and $D 1$ as described in Fig. 5, respectively, the TS drop timing via the $\mathrm{CCW}$ route at the $\mathrm{X}$-node is expressed as $x=D 2$. Then, the TS add timings via the CCW route at the X-node are expressed as $y=D 1$, respectively. Then, $z$ for the TS add timing via the CCW route at the Xnode is calculated as $z=L_{F R}-(D 1+D 2)=L_{F R}-D_{R}$. On the other hand, $z$ for the TS add timing via the $\mathrm{CW}$ route at the $\mathrm{X}$-node is calculated as $z=L_{F R}-(D 2+D 2)=L_{F R}-2 D 2$. Here, $D_{R}$ is the delay around the ring. In the case that $\mathrm{X}$ node is C-node, $D_{R}$ is set to the delay around the upper ring $\left(D_{R U}\right)$. In the case that X-node is A-node, $D_{R}$ is set to the delay around the lower ring $\left(D_{R L}\right)$. Therefore, the TS drop timing is set to the $\mathrm{X}$-node and the $D_{R}$ and $D 2$ are notified to the $\mathrm{X}$-node. These TS add timings can be calculated at the $\mathrm{X}$-node by shifting the delay time $z$ after the TS drop timing. When a link failure occurs, the proposed method dynamically changes the route of the TS transmission and TS control timing of a WDM/TDM SW and an EBC at each node according to the above TS control timings. Thanks to this mechanism, the proposed method can achieve a TS from an optical burst mode transceiver shared between any route in bufferless WDM/TDM multi-ring networks.

\subsection{Required TS Control Timings at Each Node}

Figure 6 shows the required kinds of TS control timings (TDM1-TDM8) at each node for supporting bidirectional bufferless two-stage rings network with the proposed method applied. Here, for the sake of simplicity, 
Table 1 Summarization of each parameter to set the TS control timings to the A-node.

\begin{tabular}{l|c|c|c|cc}
\hline TDM\#X & TDM\#Y & $x$ & $y$ & \multicolumn{2}{c}{$z$} \\
\hline \multirow{5}{*}{ TDM1 } & TDM2 & $D 2$ & $D 1$ & $L_{F R}-D_{R L}$ & $(: \varepsilon)$ \\
\cline { 2 - 6 } & TDM3 & $D 2$ & $D 2$ & $L_{F R}-2 D 2$ & $(: \xi)$ \\
\cline { 2 - 6 } & TDM4 & $D 2$ & $L_{F R}-D 1$ & $D_{R L}-2 D 2$ & $(: \eta)$ \\
\hline \multirow{5}{*}{ TDM5 } & TDM6 & $D 1$ & $D 2$ & $L_{F R}-D_{R L}$ & $(: \theta)$ \\
\cline { 2 - 6 } & TDM7 & $D 1$ & $D 1$ & $L_{F R}-2 D 1$ & $(: \kappa)$ \\
\cline { 2 - 6 } & TDM8 & $D 1$ & $L_{F R}-D 2$ & $D_{R L}-2 D 1$ & $(: v)$
\end{tabular}

$D 1$ : Propagation delay of right-hand route in the lower ring

$D 2$ : Propagation delay of left-hand route in the lower ring

$D_{R L}$ : Propagation delay around the lower ring $(=D 1+D 2)$

$L_{F R}$ : Length of TDM frame

the $D_{R U}$ is set to an integer multiple of the TDM frame. If $D_{R U}$ is not an integer multiple of the TDM frame, the required TS control timings for the C-node are set in the similar way for the A-node. Therefore, we focus on the $\mathrm{TS}_{\text {Sync }}$ for the A-node. First, TDM1 and TDM5 work for the CCW route and $\mathrm{CW}$ route on the upper ring, respectively. Because the same mechanism is used in left-right reversal, we focus on only how to achieve $\mathrm{TS}_{\text {Sync }}$ from the TS control timings of A-node to TDM1 on the upper ring. On the upper ring, TDM1 is used for TS add/drop at the IC-node and the $\mathrm{C}$-nodes via the $\mathrm{CCW}$. Then, each A-node is set to four kinds of TS control timings (TDM1-TDM4) to synchronize to TDM1 on the upper ring. TDM1 and TDM4 at the Anode are used for dropping the TSs, which are sent from $\mathrm{C}$ nodes via the $\mathrm{CCW}$ route and $\mathrm{CW}$ route on the lower ring, respectively. TDM2 and TDM3 at the A-node are used for TS add via the $\mathrm{CCW}$ route and $\mathrm{CW}$ route to synchronize the TS add timings at the A-node to TDM1 on the upper ring, respectively. That is, the TS add timing at the A-node via the $\mathrm{CCW}$ route is applied to TDM2 and that via the $\mathrm{CW}$ route is applied to TDM3.

In Table 1, we summarize each parameter to set the TS control timings to the A-node. As shown in Sect. 3.1, when the TDM1 as a baseline TS control timing is set to each node from the TS synchronizer via the CCW route, $z$ to start TDM2, TDM 3, and TDM 4 can be determined if the length of each route of TS transmission is measured. Here, we define $z$ for TDM2, TDM 3, and TDM4 as $\varepsilon, \xi$, and $\eta$, respectively. Then, $\varepsilon, \xi$, and $\eta$ are calculated as $L_{F R}-D_{R L}$, $L_{F R}-2 D 2$ and $D_{R L}-2 D 2$, respectively. On the other hand, TDM5-TDM8 can also be calculated in the same way for TDM1-TDM4. When the TDM5 as a baseline TS control timing is set to each node from the TS synchronizer via $\mathrm{CW}$ route, $z$ for TDM6, TDM 7, and TDM8 can be determined if the length of each route of TS transmission is measured. Here, we define $z$ for TDM6, TDM7, TDM8 as $\theta, \kappa$, and $v$, respectively. Then, $\theta, \kappa$, and $v$ are calculated as $L_{F R}-D_{R L}$, $L_{F R}-2 D 1$ and $D_{R L}-2 D 1$, respectively.

\subsection{Sequence of Proposed Method}

Here, we explain the sequence of the proposed method assuming the scenario described in Fig. 7. The assumed scenario is to execute $\mathrm{TS}_{\text {Sync }}$ at the A-node for TS control timing for the CCW route of the upper ring (TDM1). Then,

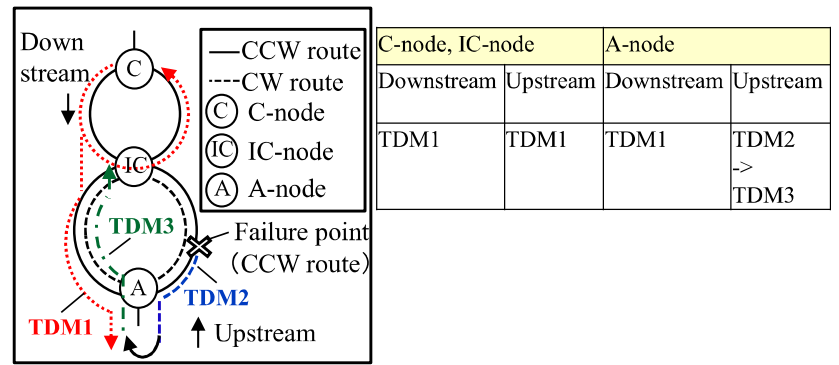

Fig. 7 Use case of required TS control timings for CCW route's failure on the lower ring.

the A-node changes the route of TS transmission and the TS control timing for sending a TS from the $\mathrm{CCW}$ route to $\mathrm{CW}$ route due to a link failure at the lower ring. In this case, the TS add timing at the A-node is changed from TDM2 to TDM3. To achieve this, the proposed method follows four steps as described in Fig. 8. The control information for achieving $\mathrm{TS}_{\text {Sync }}$ is sent using control TSs on $\lambda \mathrm{c} 1$ via the $\mathrm{CCW}$ route and $\lambda \mathrm{c} 2$ via the $\mathrm{CW}$. Control TSs on $\lambda \mathrm{c} 1$ via the $\mathrm{CCW}$ route work on TDM1 and control TSs on $\lambda \mathrm{c} 2$ via the CW route work on TDM3.

\section{(1) Setting of TDM1 (Step 1)}

The TS synchronizer in the M-node synchronizes the TDM1 for the CCW route on the upper ring between all nodes. Synchronizing the TDM1 between all nodes is achieved by sending the control packet to set the baseline TS control timing (B-packet) attached with a time stamp from the TS synchronizer to the TSCs of other nodes. This B-packet is sent using control TS via the CCW route working on TDM1. When receiving the B-packet, each TSC starts the TDM1 by starting the counter for it. This counter increments at every $F q$ at each TSC. We assume that $F q$ is synchronized among TSCs using Synchronous Ethernet [24] on the $\lambda \mathrm{c} 1$, but the phase of $F q$ is not synchronized. As a result, the start timing of the TDM1 frame at each node is delayed by the propagation delay of the $\mathrm{CCW}$.

\section{(2) Measurement of delay between each node (Step 2)}

To synchronize the required TS control timings at the Anode to the TDM1 at the IC-node, the IC-node measures the $D_{R L}$. The $D_{R L}$ is measured by subtracting the value of the counter at (a) from that at (b), where (a) is the time of receiving the B-packet at the IC-node and (b) is the time of receiving the $\mathrm{B}$-packet after going around the lower ring at the IC-node. Then, the IC-node sends measured $D_{R L}$ to the M-node using a control TS. Moreover, the M-node measures the delay from the M-node to A-node\# $j$ and IC-node using the control packet (D-packet), which is attached with a time stamp and sent from the A-node and IC-node when the Anode and IC-node receive the B-packet. This D-packet is sent using control TS via the CW route working on TDM3. However, in the initial state, A-nodes and IC-nodes transmit the D-packets at random times after receiving the B-packet from the TS synchronizer until the TDM3 is set to each 


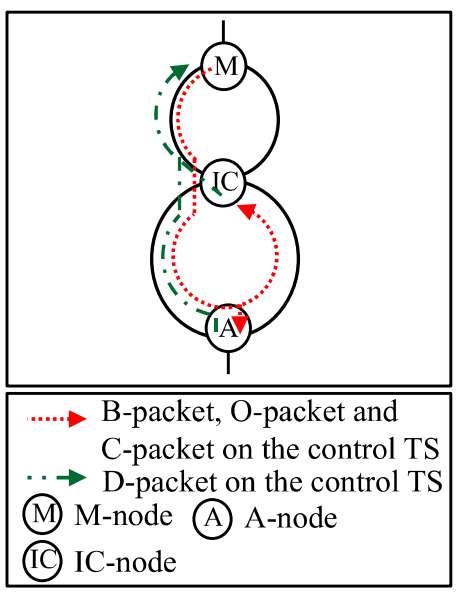

Fig. 8 Sequence of the proposed TS synchronization method.

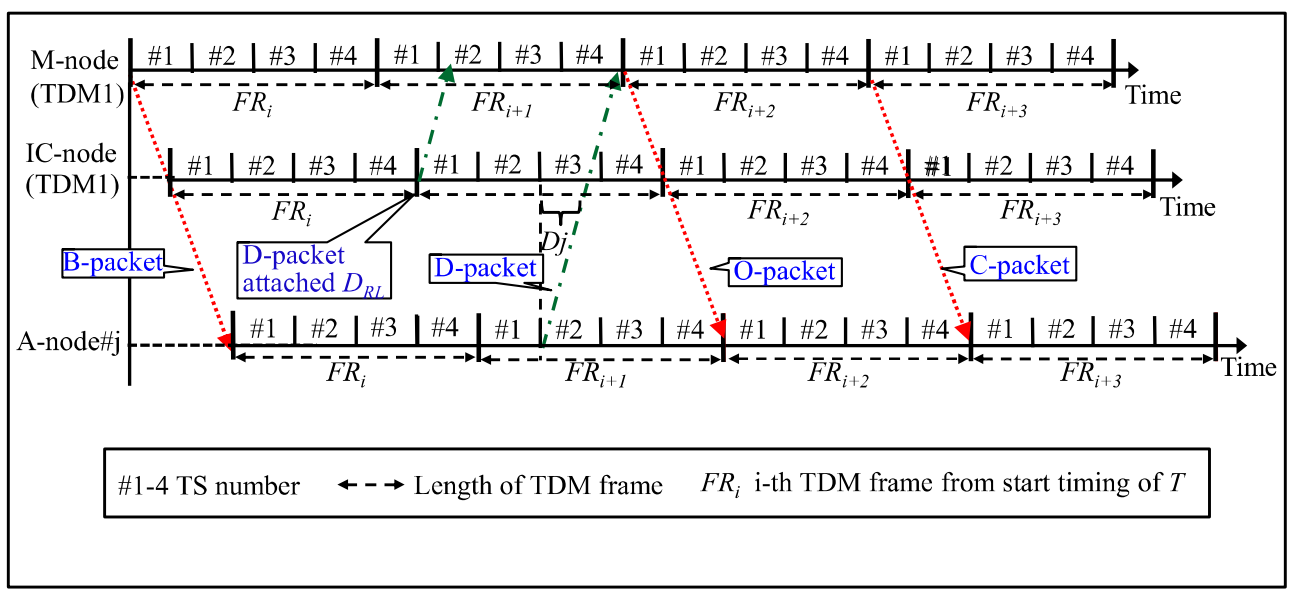

node, since TDM3 is still not being set to each node in the initial state. Then the M-node calculates the delay between the IC-node and A-node\# $j\left(D_{j}\right)$ using the above measurement values. Specifically, $D_{j}$ is calculated by subtracting the measured delay between the M-node and IC-node from the measured delay between the M-node and A-node\# $j$. Then, the M-node sets the TS control timings of TDM2, TDM3, and TDM4 to the A-node\# $j$ by starting $\varepsilon, \xi$, and $\eta$ later than the start timing of TDM1, respectively. $\varepsilon, \xi$, and $\eta$ are expressed as $L_{F R}-D_{R L}, L_{F R}-2 D_{j}$, and $D_{R L}-2 D_{j}$, respectively according to Table 1.

\section{(3) Setting of other TS control timing (Step 3)}

To start TDM2, TDM3, and TDM4 at each A-node, the ICnode sends a control packet to set other TS control timings (O-packet) attached with $\varepsilon, \xi$, and $\eta$ to each A-node. This Opacket is sent using control TS via the CCW route working on TDM1. After receiving the O-packet, each A-node starts TS control timings on TDM2, TDM3, and TDM4 using $\varepsilon$, $\xi$, and $\eta$.

The proposed method can overcome the variation in fiber delay, which is mainly due to the variation in the temperature, by executing steps $1-3$ periodically.

\section{(4) Changing route of TS transmission and TS control timing (Step 4)}

When detecting the link failure, the M-node sends a control packet to change the route of the TS transmission and TS control timing at the A-node (C-packet). This C-packet is sent using control TS via the CCW route working on TDM1. We assume that the M-node can identify the link failure depending on whether or not the C-plane's TS is received from each node. After receiving the C-packet at each A-node, the A-node changes the TS control timings and the route of the TS transmission according to information from the C-packet. Figure 9 shows the working TS control timings at the drop interface (IF) and add IF when receiving the Cpacket at the A-node. In the initial status of Fig. 9, TDM1, TDM2, and TDM3 are set and working by receiving the $\mathrm{O}-$ packet. When receiving the C-packet from the M-node, the

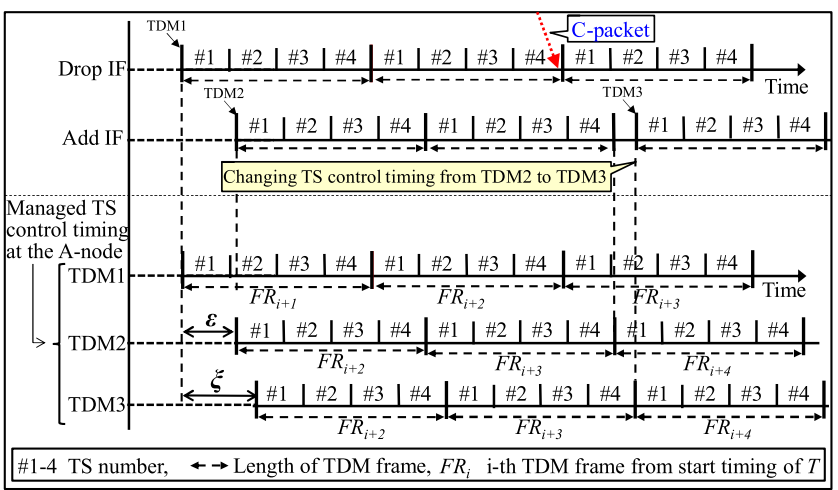

Fig.9 Working TS control timings at the drop IF and the add IF when receiving the C-packet at the A-node.

A-node changes the TS control timing at the add IF according to the information in the C-packet. In this case, the TS control timing working on the add IF is changed to TDM3 from TDM2. The timing of changing TS control timings is at the end of the TDM frame where the A-node receives the C-packet. Through this mechanism, the proposed method can share a TS from an optical burst mode transceiver between any route of arbitrary fiber length by changing both the route of the TS transmission and the TS control timing on the optical burst mode transceiver when a link failure occurs.

\subsection{Detection for the Link Failure Using Control TSs}

Here, we explain how to detect a link failure in the sequence of the proposed method as described in the previous Section. The point of how to detect a link failure in the proposed method is whether or not plural contiguous nodes detect the loss of control TSs simultaneously. This status is regarded as an occurrence of a link failure connected to the most upstream side's node in these contiguous nodes. Specifically, A-nodes and IC-nodes send control TSs attached the reception status of control TSs from the M-node. Then, Mnode not only checks the reception status of control TSs 


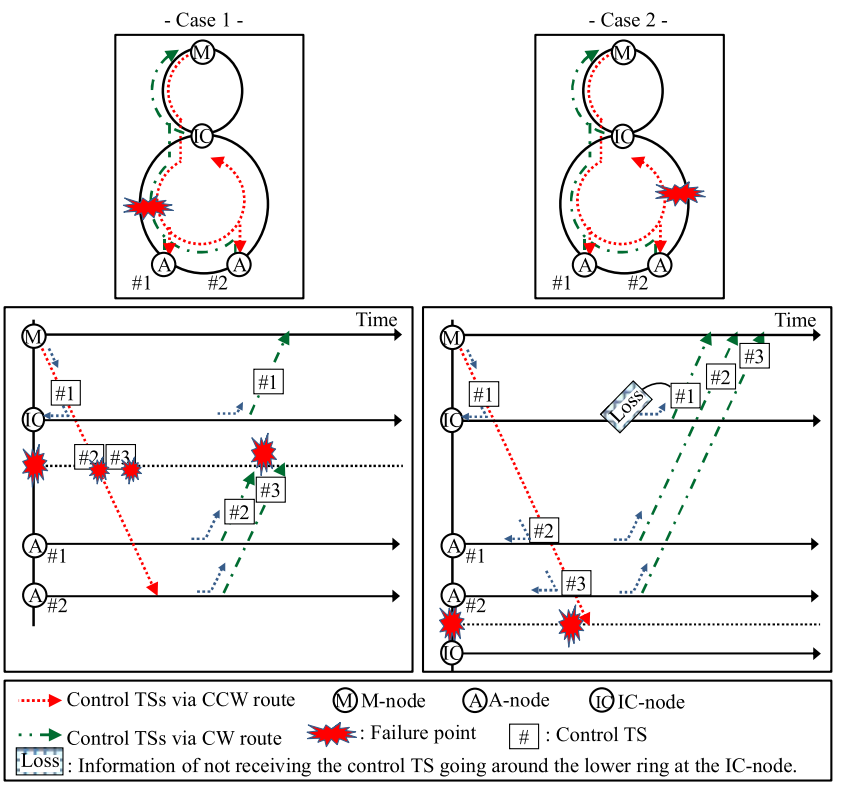

Fig. 10 Proposed method to detect a link failure.

from A-nodes and the IC-nodes but also checks the reception status of control TSs at the A-nodes and the IC-nodes. Through this mechanism, the proposed method can identify the link failure.

Following, we explain the proposed method to detect the link failure using Fig. 10, which shows a bidirectional two-stage ring network consisting of a M-node, an IC-node, and two A-nodes. IC-node, A-nodes\#1, and A-nodes\#2 are allocated control TS\#1, \#2 and \#3, respectively. Case 1 and case 2 described in Fig. 10 are available and unavailable for the detection of a link failure in the proposed method, respectively. For case 1, a link failure between IC-node and A-node\#1 occurs. On the other hand, for case 2, a link failure between IC-node and A-node\#2 occurs. In case 1, M-node cannot receive the control TSs from the plural contiguous nodes A-node\#1 and A-node\#2. Then, the M-node recognizes the occurrence of the link failure between ICnode and A-node\#1. On the other hand, in case 2, IC-node could not receive the control TS going around the lower ring. Therefore, the IC-node sends the control TS attached to the reception status of not receiving the control TS going around the lower ring from the M-node. Then M-node recognizes the occurrence of the link failure between A-node\#2 and IC-node. However, in case 2, if a failure of the optical burst mode receiver for the control TS at the IC-node occurs, the IC-node also recognizes it falsely as an occurrence of an adjacent link failure at the IC-node. Therefore, this method could not distinguish between the failure of the optical burst mode receiver for control TS at the IC-node and the link failure between IC-node and A-node\#2. There is a candidate method to address this issue, which is the deployment of two optical burst mode receivers for control TSs at the IC-node as active-active redundancy to increase the reliability of the optical burst mode receiver for control TS at the IC-node.

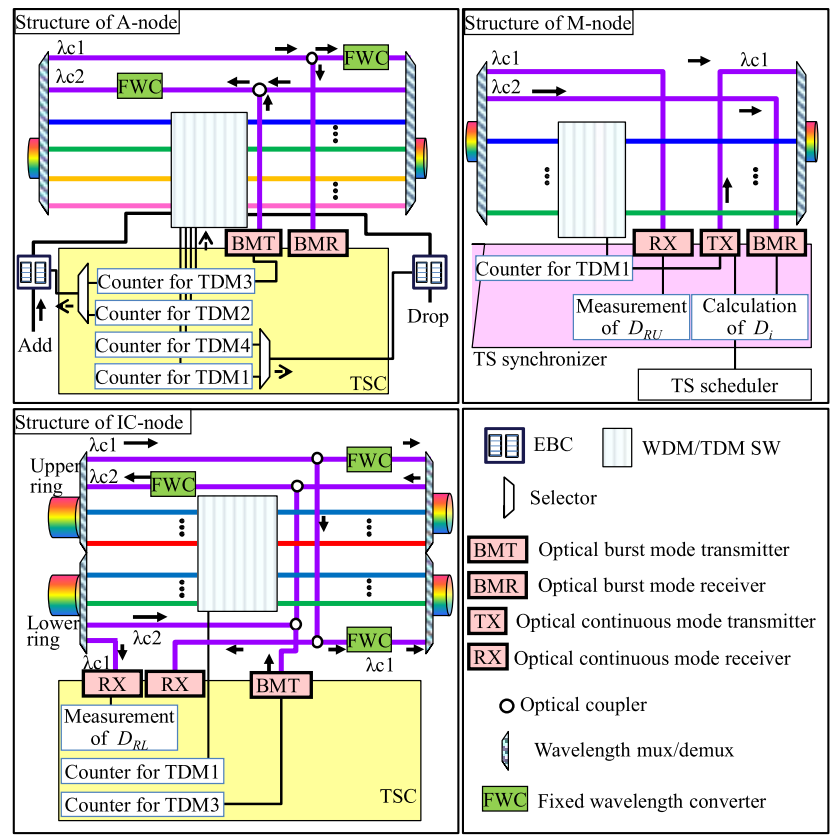

Fig. 11 Structure of each node.

\subsection{Structure of Each Node}

The structure of each node is shown in Fig. 11. This configuration is described only for supporting the CCW route on the upper ring to simplify the expression of figure. Each node has the TSC, which controls the TS control timing for a WDM/TDM SW and the EBC. To support the bidirectional routes on the upper ring, it is required that the TSC has to prepare the TS control timings for the CW route on the upper ring. Then, the EBC and WDM/TDM SW carry out the TS add/drop for each channel according to the TS control timing. Each TSC has an optical burst mode transmitter for sending the control TSs via the $\lambda \mathrm{c} 2$. The TSC at an IC-node deploys counters for TDM1 to control D-plane's TSs and TDM3 to transmit the D-packet via control TSs. Moreover, the TSCs at an IC-node and M-node deploy two receivers for the control TS to avoid collisions between the B-packet from the M-node and the B-packet after it goes around the lower ring and upper ring to measure the $D_{R L}$ and $D_{R U}$, respectively. The M-node sends and receives the control packets to and from each node through control TSs via the $\lambda \mathrm{c} 1$ and $\lambda \mathrm{c} 2$. To compensate for the power loss of the $\lambda \mathrm{c}$, a fixed wavelength converter (FWC) is set to each port of the wavelength filter for the $\lambda \mathrm{c} 1$ and the $\lambda \mathrm{c} 2$.

In contrast, the A-node has add/drop interfaces connected to the EBC. The EBC and WDM/TDM SW interface at the A-node have to work at TDM1, TDM2, TDM3, and TDM4 according to the route of allocated TS transmission. To control the EBC and WDM/TDM SW's interface, the TSC at the A-node deploys the counters for TDM1, TDM2, TDM3, and TDM4. Then, according to the instructions from the M-node, the TSC changes the TS control timing 
of the EBC and WDM/TDM SW's interface by switching the selector connected to each counter.

Here, we described how to realize the FWC and a candidate method to construct the FWC cost-effectively. As shown in Fig. 11, a FWC has to receive control TSs both from a FWC at an adjacent node and from its own node. Therefore, the FWC has to implement an optical burst mode receiver. Thus, a FWC consists of an optical burst mode receiver and a fixed-wavelength laser. In terms of costeffectiveness for the FWC, there is further study for the construction of the FWC. However, as a candidate method to construct the FWC at a low cost, there is a method to apply a continuous mode receiver to the FWC. This concept is shown in [25]. This is achieved by adjusting the output power of an optical burst mode transmitter for the control TS at each node. This leads to the possibility of the application of a continuous mode receiver to a FWC. This means that further reduction of the CAPEX for the FWC may possibly be achieved in comparison to that for the FWC with application of an optical burst mode receiver.

\subsection{Structure of WDM/TDM SW}

Figure 12 shows the configuration of the WDM/TDM SW. The WDM/TDM SW has to execute TS add/drop operations for any route, any channel, and any TS. To achieve this, a WDM/TDM SW is composed of tunable wavelength converters (TWCs) at each input port and a wavelength filter. The WDM/TDM SW changes the route of TSs transmission by changing the wavelength of TS at the TWC, which can change the wavelength for every TS [12]. The TWC is controlled by the TSC based on the information of TSA and TS control timings set by M-node. A merit of the application of TWC is its ability to convert the wavelength to another one. This can avoid TS fragmentations and increase the efficiency of TS utilization [26].

Here, we show the implementation for the TWC. A practical way to directly convert wavelength at the optical level has not yet been established. Therefore, we use the TWC based on Optical-Electrical-Optical (OEO) conversion. In our approach, a TWC consists of a high-speed tunable laser and an optical burst mode receiver [12]. This optical burst mode receiver can recover the phase shift and loss imbalance for TSs from an adjacent node. This loss imbalance is caused by the combinations between the input

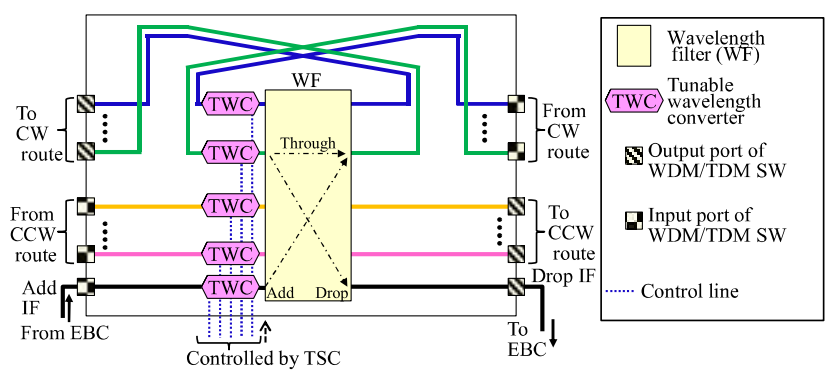

Fig. 12 Structure of WDM/TDM SW. port and output port in the wavelength filter [27]. In this case, the TWC receives optical burst signals, which it converts to an electrical signal, and then transmits optical burst signals on the specified wavelength for every TS. Therefore, a TWC based on OEO conversion has to be deployed for each port of the WDM/TDM SW. As our previous study, we show our way of thinking for the CAPEX of this TWC [18]. In the reference to [18], to achieve the cost-effectiveness of the OL2SW-NW as the whole network system compared to the OTN as a conventional technology, the target value of the cost of TWC should be equal to the 3R regenerator of OTN. This $3 \mathrm{R}$ regenerator performs signal recovery by reamplifying, reshaping, and retiming the signal at the OEO processing. Currently, the tunable laser is still expensive. However, thanks to the progress of research and development of TDM/WDM-PON [28], which tunable lasers are applied for, tunable lasers will gradually come down in price.

\section{Accuracy and Scalability for Proposed $T S_{\text {Sync }}$ Method}

\subsection{Accuracy of Proposed $\mathrm{TS}_{\mathrm{Syn}}$ Method}

We estimate the accuracy of $\mathrm{TS}_{\text {Sync }}\left(T S_{a c c}\right)$ applied to the proposed method. This influences the effective bandwidth. Figure 13 defines $T S_{a c c}$ as the difference between the TS control timing at each node and the arrival timing of the TS. Therefore, the length of a guard band $\left(L_{G B}\right)$ has to be set to be more than $T S_{a c c}$ to prevent TS collisions. The $T S_{a c c}$ consists of two factors: fluctuation of TS control timing and chromatic dispersion [18].

\section{(1) Fluctuation of TS control timing}

There are two factors that affect the fluctuation of TS control timing: (A) the processing of receiving and sending the control TS and (B) difference in the length of C-plane's and D-plane's route in a node $(\gamma)$. (A) consists of (A1), which is the difference in the phase of $F q$ between each node $(\alpha)$, and (A2), which is $64 \mathrm{~B} / 66 \mathrm{~B}$ encoding of the $10 \mathrm{G}-\mathrm{EPON}$ [7] $(\beta)$. (A1) is attributed to the difference between the arrival timing of the TS and the phase of $F q$ at the receiver. (A2) is attributed to the speed-conversion process of $64 \mathrm{~B} / 66 \mathrm{~B}$ encoding, which transforms between 64-bit word at $161 \mathrm{MHz}$ and 66-bit word at $156 \mathrm{MHz}$. Here, we discuss the degree of (A) and (B) for $T S_{a c c}$ at each step described in Sect. 3.3. We define $N_{L}, N_{U}$, and $N_{M}$ as the number of A-nodes on a lower ring, $\mathrm{C}$-nodes, and IC-nodes, respectively.

Step 1 is affected by (A) and (B) when sending and receiving the B-packet. (A) is generated by (A1) for receiving the B-packet and (A2) for sending/receiving the B-packet. Hence, (A) becomes $\alpha+2 \beta$. Meanwhile, (B) is generated at

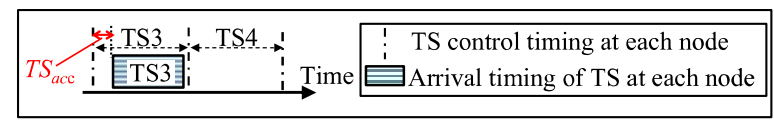

Fig. 13 Definition of $T S_{a c c}$. 
every node the B-packet goes through. Then, (B) becomes $\gamma\left(N_{U}+N_{M}+N_{L}\right)$ at maximum.

Step 2 is affected by (A) and (B) when receiving the B-packet twice at the IC-node to measure $D_{R L}$ and sending/receiving the D-packet between the A-nodes and $\mathrm{M}$ nodes. For the B-packet, (A) is generated by (A1) and (A2) at the IC-node receiving the B-packet twice to measure $D_{R L}$. Hence, (A) and (B) for B-packets become $2 \alpha+2 \beta$ and $\gamma N_{L}$, respectively. (A) and (B) for D-packets are the same as those in Step 1. Hence, (A) and (B) for D-packets become $\alpha+2 \beta$ and $\gamma\left(N_{U}+N_{M}+N_{L}\right)$ at maximum. Note that Steps 3 , which are the processes of setting TDM 2, TDM3, and TDM4 using the O-packet, do not affect $T S_{a c c}$ because TDM 2, TDM3, and TDM4 are set based on TDM1 at the A-node regardless of the arrival timing of the O-packets.

(2) Chromatic dispersion

In the assumed network model, it is impossible to ignore the effects of chromatic dispersion because the total length of fiber becomes several hundred kilometers at maximum. The delay time for each channel affected by the chromatic dispersion is described as $D_{d} \cdot W \cdot G_{r} \cdot L_{\text {total }}$; here, $D_{d}$ is the chromatic dispersion, $W$ is the number of wavelengths for a fiber, $G_{r}$ is the channel spacing, and $L_{\text {total }}$ is the maximum distance of a TS traversal. Then, $T S_{a c c}$ is expressed as Eq. (1).

$$
\begin{aligned}
T S_{a c c}= & (4 \alpha+6 \beta)+2 \gamma\left(\frac{3}{2} N_{L}+N_{M}+N_{U}\right) \\
& +D_{d} B_{f i b e r} G_{r} L_{\text {total }}
\end{aligned}
$$

As described in Sect. 3.3, the method to measure the propagation delay between each node is using control TSs. However, a difference occurs between the propagation delay for control TS and that for the desired data channel affected by chromatic dispersion. There are two methods to deal with this difference in propagation delay. One is to adjust the TS control timings according to the propagation delay of TS on each data channel. In this method, each TS control timing is corrected by calculating $D_{d} \cdot \Delta \lambda \cdot L_{\text {total }} ; \Delta \lambda$ is the difference of wavelength between the control channel and the desired data channel. This approach can increase in the effective bandwidth caused by the achievement of the reduction of the guardband. However, this approach is required for adjusting TS control timings at every TS according to the TS's wavelength. Therefore, it leads to difficulty in controlling of TSs and the increase in the required hardware resources in the TSC. Another method is, in terms of the simplification for the implementation of the TSC, setting the guardband for absorbing the difference of the propagation delay between the control channel and the desired data channel to each TS. In this paper, we adopt the latter on grounds of simplification for the implementation of the TSC.

\subsection{Scalability for Proposed $\mathrm{TS}_{\text {Sync }}$ Method}

Following, we show the scalability for the number of stages and rings in the proposed method. Regarding the number of stages, the greater the number of stages $\left(: N_{\text {Stage }}\right)$ increase, the more the number of routes of TS transmission (: $\left.N_{\text {Route }}\right)$ increases according to $N_{\text {Route }}=N_{\text {Stage }} \hat{2}$. This means that the required number of TS control timings and wavelengths increase in proportion to $N_{\text {Route }}$. For the number of TS control timings, this affects the size of the TS table in the TSC to manage the TS control timings and the information of allocated TSs. Therefore, the more the number of stages increases, the more the required hardware resources in TSCs increases. Therefore, it is necessary to design the size of TS table in the TSC in accordance with the number of accommodated stages in the target network system. On the other hand, the increase in the number of rings leads to an increase in the number of IC-nodes. Therefore, the $T S_{a c c}$ deteriorates caused by increasing in the number of accommodated nodes which TS goes through. Therefore, it is necessary to design the effective bandwidth in accordance with the number of accommodated rings for the target network systems.

\section{Characteristic of $N_{I F}$ for OL2SW-NW}

There is a probability of deteriorating $T S_{a c c}$ caused by $\mathrm{TS}_{\text {Sync }}$ for the whole network. Therefore, $L_{G B}$ must be set at more than $T S_{a c c}$ for every TS to avoid TS collisions. That is to say, the larger $L_{G B}$ is, the more $N_{I F}$ increases under the condition that the volume of accommodated traffic is the same. In the following, we evaluate the required $L_{G B}$ of the OL2SW-NW in the assumed network model and the $N_{I F}$.

\subsection{Assumed Network Parameters}

To evaluate $L_{G B}$, here we show the assumed network parameters. Figure 14 shows the assumed physical and logical network model of the OL2SW-NW, which is a bidirectional two-stage ring network aggregating traffic from $1000 \mathrm{SWs}$ on the access network to 10 IP routers on the core network. This means that the OL2SW-NW accommodates 1000 Anodes and $10 \mathrm{C}$-nodes. We assume that the maximum number of $N_{L}$ is 25 . In this case, $N_{M}$ becomes 40 at maximum. The fiber lengths around the upper ring and the lower ring are 20 and $400 \mathrm{~km}$, respectively. Also, we assumed that one L2SW has a $10 \mathrm{GE}$ interface that accommodates 1000 users. Since the assumed calling rate is $10 \%$ at most, 100 users can simultaneously connect to the OL2SW-NW from

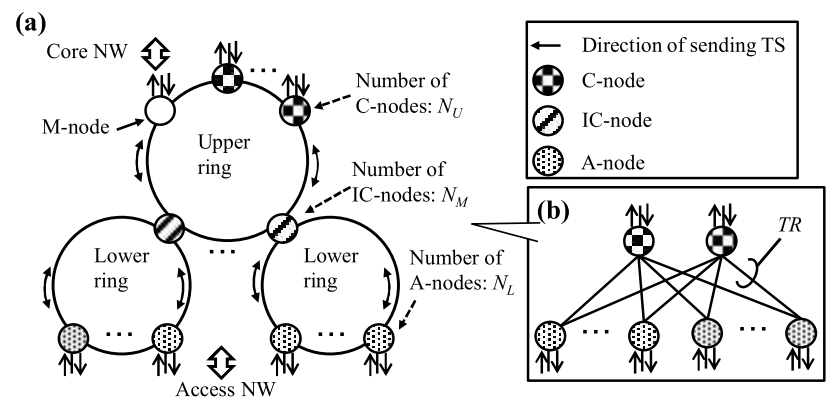

Fig. 14 Assumed network model; (a) physical network and (b) logical network. 
an L2SW. Also, we assume that the average traffic from a user flows at $50 \mathrm{Mbps}$. Then, the total average traffic from an A-node becomes $5 \mathrm{Gbps}$. In this case, the traffic volume on average from one L2SW to a C-node $(T R)$ becomes 0.5 Gbps. Other network parameters for evaluating $L_{G B}$ are shown as follows. We set $\gamma$ to be $1 \mathrm{~m}$ (equal to $5 \mathrm{~ns}$ ), and $\alpha$ and $\beta$ were 6.4 ns assuming that $F q$ was $156 \mathrm{MHz}$. Also, we assumed $G_{r}, W$, and $D_{d}$ were $50 \mathrm{GHz}, 100$ wavelengths, and $17 \mathrm{ps} / \mathrm{nm} / \mathrm{km}$ [29], respectively. Here, the $T R$ was set at from $0.1 \mathrm{Gbps}$ to $1 \mathrm{Gbps}$. In other words, the maximum amount of traffic from one L2SW was set to $1 \mathrm{Gbps}$ or 10 Gbps, respectively.

Here, we described the required interface speed for the control channel. The OL2SW-NW has to both change the route of TS transmission when the link failure occurs and control the DBA. Therefore, it is desirable for the achievement of both the fast restore from the occurrence of the failure and shortened DBA cycle to increase the efficiency of the accommodated traffic [13]. The control information related to DBA and the detection of a link failure is transmitted using control TSs between each node. As an example, the size of control information for DBA becomes about 10 Kbytes in the assumed network model according to [13]. If the interface speed for the control plane is $1 \mathrm{Gbps}$, the required size of a control TS become about $100 \mu \mathrm{s}$. In this case, the time to collect control information from 1000 Anodes become about $100 \mathrm{~ms}$. This value not only affects the effective bandwidth to accommodate the traffic in terms of DBA, but also has a significant impact on the required time to restore a link failure. In this case, our proposed method isn't sufficient for conventional ether ring protection to provide 50-ms protection [30]. Therefore, we adopt $10 \mathrm{Gbps}$ interface speed for the control plane.

\subsection{Characteristic of P-OADM and OL2SW-NW}

Here, we show the characteristic of $N_{I F}$ in the OL2SWNW compared with that of the P-OADM as a conventional technology (Table 2). We define $N_{I F}$ in the OL2SW$\mathrm{NW}$ as $N_{I F}^{O L 2}$ and in the P-OADM as $N^{P-O A D M}{ }_{I F}$. We show the difference in characteristics between P-OADM and OL2SW-NW in Table 2.

P-OADM extracts the control information from a packet and transports the control information via a control channel so as not to have to demodulate all the wave-

Table 2 Comparison of P-OADM and OL2SW-NW.

\begin{tabular}{|c|c|c|}
\hline & P-OADM & OL2SW-NW \\
\hline $\begin{array}{l}\text { Packet buffers } \\
\text { and optical IF } \\
\text { in } \\
\text { intermediate } \\
\text { node }\end{array}$ & $\begin{array}{l}\text { [Poor] Need for packet buffers } \\
\text { and optical IF in intermediate } \\
\text { node to insert optical slots to } \\
\text { packet buffers to avoid } \\
\text { collision of TS between rings. }\end{array}$ & $\begin{array}{l}\text { [Fair] No need to set packet } \\
\text { buffers in intermediate node by } \\
\text { synchronizing TSs in all nodes. }\end{array}$ \\
\hline $\begin{array}{l}\text { Effective } \\
\text { bandwidth } \\
\left(B U_{e f f}\right)\end{array}$ & $\begin{array}{l}\text { [Fair] Guard band attributed to } \\
\text { the } T S_{a c c} \text { for whole network not } \\
\text { required. }\end{array}$ & $\begin{array}{l}{[\text { Poor }] \text { Decreasing because of }} \\
\mathrm{TS}_{\text {Sync }} \text { in whole network. }\end{array}$ \\
\hline $\begin{array}{l}\text { Fiber } \\
\text { adjustment }\end{array}$ & $\begin{array}{|lr|}\text { Required] For the fibers } \\
\text { between bidirectional routes } \\
\text { and between multi rings. }\end{array}$ & [Not required] \\
\hline
\end{tabular}

lengths at each node. According to this control information, each node autonomously operates the transmission of optical slots. The control information also provides the timing of optical slot transmission to an adjacent node. Then, the size of $L_{G B}$ for the P-OADM is equal to the difference in the length of the C-plane's and D-plane's route in a node. Therefore, the P-OADM can increase the effective bandwidth. However, it is required to adjust the fiber between each node to support a bidirectional multi-ring network. These fiber adjustments are required for the interconnection of rings to achieve $\mathrm{TS}_{\text {Sync }}$ between rings and for the link between each node to achieve $\mathrm{TS}_{\text {Sync }}$ between bidirectional routes. Moreover, the IC-node of the P-OADM requires the packet buffer because it is not a guarantee of optical slot collision between the upper and lower ring. If there is no contention from other incoming optical slots, the optical slot is switched to the output port, passing through the IC-node transparently. However, in the case of contention, the packets in the optical slot are stored to the shared packet buffers for temporary storage. Therefore, if $T R$ is larger, $N^{P-O A D M}{ }_{I F}$ at the IC-node is increased.

On the other hand, the OL2SW-NW can multiplex the traffic without applying an electrical L2SW for the whole network. This is achieved by applying $\mathrm{TS}_{\mathrm{Sync}}$ for the whole network and controlling DBA. Thanks to this mechanism, the OL2SW-NW does not need the packet buffer at the ICnode. As a result, it is possible to reduce $N^{O L 2}{ }_{I F}$ to insert the packet buffer at the IC-node. In contrast, the OL2SW-NW requires the increase in $L_{G B}$ to avoid TS collisions for the $\mathrm{TS}_{\text {Sync }}$ for the whole network. Therefore, a slight decrease in effective bandwidth occurs.

\subsection{Mathematical Expression for $N_{I F}$ at Each Method}

Here, we explain the mathematical expressions for $N_{I F}$ at each method. We show the model of $N^{\text {tech }}{ }_{I F}$ when using the technology tech $\in\{\mathrm{OL} 2, \mathrm{P}-\mathrm{OADM}\} . \quad N^{\text {tech }}{ }_{I F}$ for each method is expressed as

$$
\begin{aligned}
& N_{I F}^{\text {tech }}=\sum_{i \in S}\left[\sum_{j \in D}\left[\left(T_{i, j} / B_{T S}\right) / B U_{e f f}^{\text {tech }}\right] / N_{T S}\right\rceil \\
& +\sum_{j \in D}\left\lceil\sum_{i \in S}\left[\left(T_{i, j} / B_{T S}\right) / B U_{e f f}^{\text {tech }}\right] / N_{T S}\right]+N_{M} N_{I F-I C}^{\text {tech }}
\end{aligned}
$$

Here, \lceil\rceil represents the ceiling function, $S$ is the node sending the traffic, $D$ is the node receiving the traffic from $S, T$ is a matrix indicating the traffic, $T_{i, j}$ is the traffic from node $i$ to node $j, B U^{\text {tech }}$ eff is the effective bandwidth for allocated TSs, $B_{T S}$ is the capacity of a TS expressed in bps, $N^{\text {tech }}{ }_{I F_{I} I C}$ is the $N_{I F}$ at an IC-node, and $N_{T S}$ is the number of TSs on a wavelength in a $L_{F R}$. $B U^{\text {tech }}{ }_{\text {eff }}$ is expressed as $\left(N_{A-T S} \cdot L_{T S}-L_{G B}^{\text {tech }}\right) /\left(N_{A-T S} \cdot L_{T S}\right) . N_{A-T S}$ and $L_{G B}^{\text {tech }}$ are the number of allocated TSs for a logical path and the length of the guard band for a TS with the application of tech $\in\{\mathrm{OL} 2, \mathrm{P}-\mathrm{OADM}\}$, respectively. $N_{A-T S}$ increases in proportion to the accommodated $T R$. The first, second, and 
third terms of Eq. (2) denote $N_{I F}$ at the source node, $N_{I F}$ at the destination node, and $N_{I F}$ at the IC-node, respectively. This formulation has two kinds of ceiling functions. The outer ceiling function represents the unit of an optical interface to accommodate the TSs. The inner ceiling function represents the unit of TS. The difference in $B U^{\text {tech }}{ }_{\text {eff }}$ between the P-OADM and OL2SW-NW is $L^{\text {tech }}{ }_{G B}$ caused by $T S_{a c c}$. The $T S_{a c c}$ of the OL2SW-NW is deteriorated in order to require $\mathrm{TS}_{\mathrm{Sync}}$ for the whole network. However, the OL2SW-NW does not require $N_{I F}$ to avoid TS collisions at the IC-node because of the achievement of $\mathrm{TS}_{\text {Sync }}$ for the whole network.

\section{Evaluation of Proposed Method \& Discussion}

We evaluated the effectiveness of our proposal from two perspectives; the first is (i) the feasibility of the proposed method, which changes TS control timing dynamically. The index of evaluation (i) is the time of changing TS control timing and $T S_{a c c}$ using prototype systems. These have an effect on the size of $B U_{e f f}$, which affects the $N_{I F}$. The second is (ii) the evaluation of the required $N_{I F}$ assuming the results of (i). This affects the CAPEX.

\subsection{Experimental \& Evaluation Conditions}

\section{(1) Experimental conditions for changing TS control timing and $T S_{\text {acc }}$}

We conducted an experiment on TS exchange in a bidirectional bufferless WDM/TDM two-stage rings network using the prototype systems. We evaluated the speed of changing TS control timings $\left(T_{\text {timing }}\right)$ and $T S_{a c c}$. The experimental setup for evaluating $T_{\text {timing }}$ and $T S_{a c c}$ is shown in Fig. 15. We set a M-node, an IC-node, and two A-nodes and set TSCs for these nodes. The scenario is that the A-node\#1 sent a TS via the CCW route to the IC-node. Then, the A-node\# 1 changed the route of the TS transmission and TS control timing when a link failure between the A-node\#1 and the A-node\#2 occurred (Fig. 15(b)). We emulated a TWC in the WDM/TDM SW using both a $1 \times 2$ optical SW [31] and optical couplers. Figure 15 (c) shows the prototype of TSC. This prototype of TSC has a $10 \mathrm{G}$ optical burst mode transmitter for the control plane and a 1GE IF for connecting to the EBC for the control plane. This TSC also connects to the $1 \times 2$ optical SWs using coaxial cables (Fig. 15(a)). If the TSC receive the C-packet from the TS synchronizer, the TSC send both the signal of changing the switched port to the $1 \times 2$ optical SW connected to the add IF via a coaxial cable and the signal of changing the TS control timing to the EBC via the 1GE. We used three wavelengths, one for the data channel $(\lambda 1)$ and two control channels $(\lambda \mathrm{c} 1, \lambda \mathrm{c} 2)$. To confirm that the TSs from an EBC are shared between the $\mathrm{CW}$ route and $\mathrm{CCW}$ route and do not collide at the IC-node, a real-time oscilloscope was set to the IC-node's interface. The TS numbers allocated to A-nodes\#1 and \#2 were TS\#1 and TS\#3. Moreover, to measure $T S_{a c c}$ while changing the TS control timing for an arbitrary fiber length between each

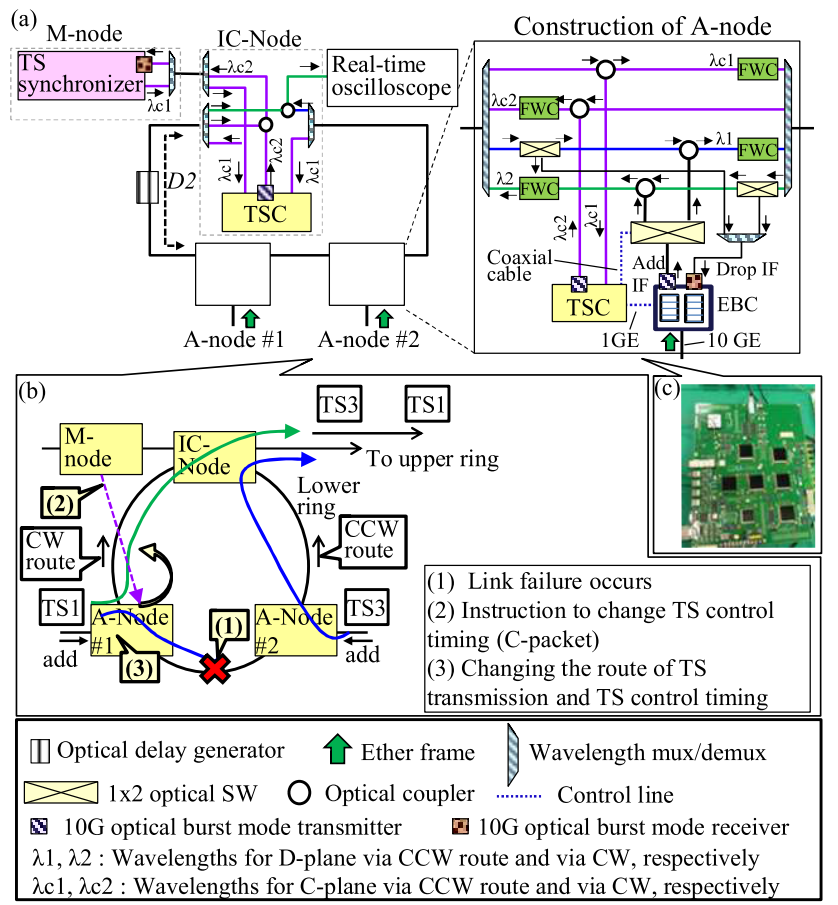

Fig. 15 Experimental conditions; (a) experimental setup, (b) experimental scenario, and (c) prototype of TSC.

node, we set the optical delay generator for the length between the IC-node and A-node\#1 (D2).

\section{(2) Simulation conditions for required $N^{O L 2}{ }_{I F}$}

To confirm the effectiveness of the OL2SW-NW, we evaluated $N_{I F}^{O L 2}$ on the basis of the experimental results and Eq. (2). Here, the $T R$ described in Fig. 14 was set to three patterns: $0.1,0.5$, and $1 \mathrm{Gbps}$ according to the assumed network parameters in Sect. 5.1. In other words, the amount of traffic from one L2SW was set to 1,5 , and $10 \mathrm{Gbps}$, respectively. Here, we define the ratio of $N_{I F}\left(R_{I F}\right)$ as $N^{O L 2}{ }_{I F} / N^{P-O A D M}{ }_{I F}$. If $R_{I F}$ is smaller than 1 , this means that $N^{O L 2}{ }_{I F}$ can be reduced more than $N^{P-O A D M}{ }_{I F}$ when accommodating the same amount of traffic. When multiple TSs were allocated for the same destination, we applied a multi TS bonding technique [32], which allocates successive TSs. $L_{F R}, L_{T S}$ and $L_{G B}$ were $1 \mathrm{~ms}, 10 \mu \mathrm{s}$, and $100 \mathrm{~ns}$, respectively. To evaluate the applicable area for the OL2SW-NW, we changed $N_{L}=1-25, N_{M}=5,40$, and $T R=0.1,0.5$, $1 \mathrm{Gbps}$, respectively.

\subsection{Experimental Results of Speed of Changing TS Con- trol Timings}

Figure 16 shows the results of $T_{\text {timing }}$ at A-node\#1, which changed the TS control timing from TDM2 to TDM3. As you can see, the proposed method could achieve $T_{\text {timing }}$ within one TDM frame $(1 \mathrm{~ms})$ from receiving the C-packet. This switching time is not the constraint of hardware specifications of TSC but the TS control timing that TSC switches to. This is determined by the difference in the delay between 


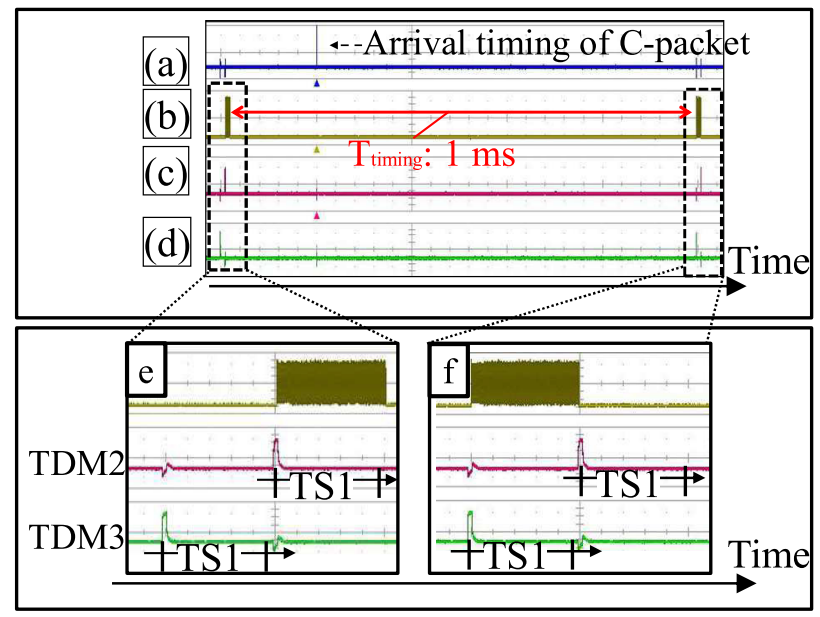

Fig. 16 D-plane's TS signals from A-node \#1 with changing TS control timings; (a) arrival timing of C-packet at A-node \#1, (b) D-plane's TS signals with changing TS control timing from A-node \#1, (c) TS control timing for add via CCW route (TDM2), (d) TS control timing for add via CW route (TDM3) at A-node \#1, and (e, f) enlarged view of (b), (c), and (d).

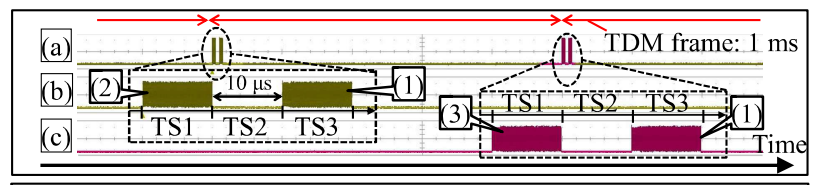

D-plane's TS signal from

(1) A-node \#2 via CCW route

(2) A-node \#1 via CCW route

(3) A-node \#1 via CW route

Fig. 17 D-plane's TS signals from each A-node changing both routes of TSs transmission and TS control timings at IC-node; (a) D-plane's TS signals from different A-nodes, and (b, c) enlarged view of (a).

the routes of TS transmission. However, the time taken to identify a link failure was not taken into account in this experiment. This time is determined by the network size. If the $D_{R}$ is several hundred $\mathrm{km}$, the time taken to detect the link failure at the M-node is about the round trip time between an A-node and M-node. Therefore, this time becomes a few ms. Hence, this result could be sufficient for conventional ether ring protection to provide 50-ms protection [30]. Figure 17 shows the results of receiving the D-plane's TS signals at the IC-node from different A-nodes by changing both the route of the TS transmission and the TS control timing at $D 2=2 \mu$ s for the above experiment. As you can see, these signals were multiplexed without TS collisions according to the TS control timing at the IC-node.

\subsection{Experimental Results of $T S_{a c c}$}

Figure 18 shows the $T S_{a c c}$ with the proposed method at the IC-node for the length of $D 2$ under the same conditions for the above experiment. It shows the maximum, minimum, and average values for 10 seconds measurement time at length $D 2$ from 1 to $10 \mu \mathrm{s}$. At each length of $D 2$, the width of the $T S_{a c c}$ means the fluctuations of TS control timings caused by $64 \mathrm{~B} / 66 \mathrm{~B}$ encoding of the 10G-EPON described

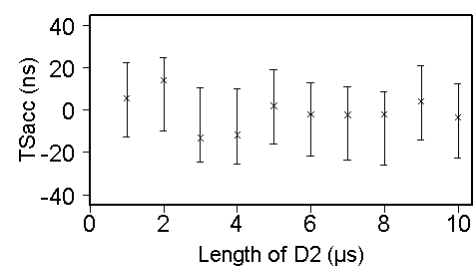

Fig. $18 T S_{a c c}$ with proposed method.

in Sect. $4.1 ; 6 \beta$ (= $38.4 \mathrm{~ns}$ at maximum). The difference in the offset of the $T S_{a c c}$ between each length of $D 2$ means the fluctuations of TS control timings caused by the difference in the phase of $F q$ between each node described in Sect. 4.1; $4 \alpha(=25.6 \mathrm{~ns}$ at maximum $)$.

In total, the $T S_{a c c}$ were within $60 \mathrm{~ns}$ of the length of $D 2$. This result was almost equal to the designed value for the fluctuation in TS control timing: $4 \alpha+6 \beta$ in the first term of the right part of Eq. (1) at $\alpha, \beta=6.4$ ns without considering both the affection of the chromatic dispersion and the difference in the length of C-plane's and D-plane's route for all accommodated nodes.

Based on these experimental results, we evaluated the total $T S_{a c c}$ in the proposed method considering the assumed network parameters described in Sect. 5.1. Firstly, the maximum difference in the length of C-plane's and D-plane's routes in the whole network was calculated as $375 \mathrm{~ns}$ by considering the total number of accomodated nodes according to the description in Sect. 4.1 (1). Next, the maximum difference in the propagation delay for each channel affected by the chromatic dispersion was calculated as $286 \mathrm{~ns}$ considering the length of the ring according to the description in Sect. 4.1 (2). As a result, $T S_{a c c}$ becomes about $720 \mathrm{~ns}$ in total. This is equal to $92.8 \%$ of the effective bandwidth for $L_{T S}(: 10 \mu \mathrm{s})$. In the following, we evaluate the required $N_{I F}$ based on this effective bandwidth.

\subsection{Simulation Results of $N_{I F}$}

Finally, we evaluate the required $N_{I F}$ for each method by calculating $R_{I F}$ (: $\left.N^{O L 2}{ }_{I F} / N^{P-O A D M}{ }_{I F}\right)$ using Eq. (2). Figure 19 shows the $R_{I F}$ when changing $N_{L}$ (from 1 to 25 ), $N_{M}$ (: 5 and 40) and $T R$ (: $0.1,0.5$, and $\left.1 \mathrm{Gbps}\right)$, respectively. It is clear that the $R_{I F}$ decreased as the $T R$ increased. This is because, for the P-OADM, the larger the $T R$ increases, the larger the number of collisions of TSs increases at the IC-node. Therefore, for the P-OADM, the required packet buffer and $N_{I F}$ in the IC-node increase according to the increase in $N_{L}$ and $N_{M}$. In comparison, for the OL2SW$\mathrm{NW}, B U_{\text {eff }}$ increases as a result of the successive TSs allocations [32] according to the increase in $T R$. However, as a trade-off relationship, $N^{O L 2}{ }_{I F}$ increases according to $N_{M}$ and $N_{L}$ because the OL2SW-NW requires the increase in $L_{G B}$, which is proportional to $N_{M}$ and $N_{L}$. As a result, $R_{I F}$ increases according to the increase in $N_{M}$ and $N_{L}$.

From the above results, we found that the OL2SW-NW was effective in terms of the reduction of $N_{I F}$. In particular, 


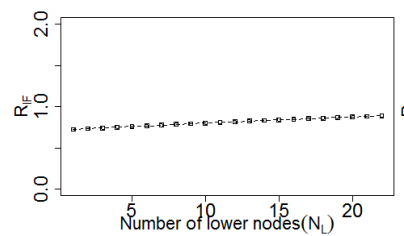

(a) $N_{M}=5, T R=0.1 \mathrm{Gbps}$

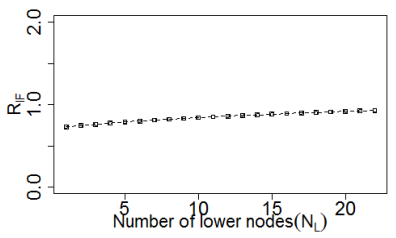

(c) $N_{M}=5, T R=0.5 \mathrm{Gbps}$

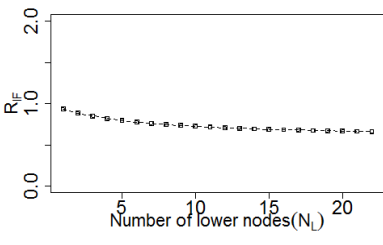

(e) $N_{M}=5, T R=1 \mathrm{Gbps}$

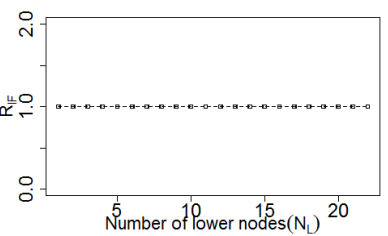

(b) $N_{M}=40, T R=0.1 \mathrm{Gbps}$

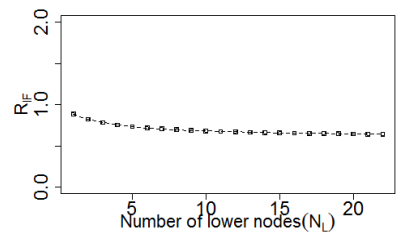

(d) $N_{M}=40, T R=0.5 \mathrm{Gbps}$

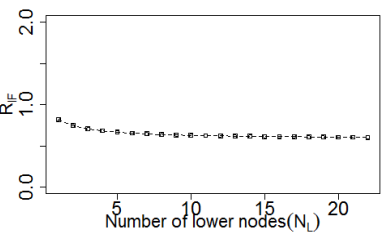

(f) $N_{M}=40, T R=1 \mathrm{Gbps}$
Fig. $19 R_{I F}$ when changing $N_{M}(5$ or 40$)$ and $N_{L}$ (from 1 to 25 ) at $T R=$ $0.1,0.5,1$ Gbps.

in this experimental condition, $R_{I F}$ at minimum became 0.7 at $N_{L}=25, N_{M}=40$, and $T R=1 \mathrm{Gbps}$. This means the OL2SW-NW could reduce the $N_{I F}$ by $30 \%$ at maximum. In view of the above results, we could find that the OL2SWNW could be effectively applied to an aggregation network, which has to accommodate an ever-growing traffic volume while suppressing the CAPEX of aggregation networks.

\section{Conclusion}

In this paper, we proposed an OL2SW-NW for supporting bidirectional bufferless multi-ring networks to achieve a cost-effective and reliable aggregation network toward the future internet. The proposed network can eliminate both the packet buffer and optical interface at the IC-node by achieving $\mathrm{TS}_{\text {Sync }}$ for the whole network in the arbitrary lengths and arbitrary routes. To achieve this, we proposed a $\mathrm{TS}_{\text {Sync }}$ method that dynamically changes the routes of TS transmission and the TS control timing of a WDM/TDM SW and an EBC at each node according to the occurrence of a link failure. The important point of the OL2SW-NW is how to decrease $L_{G B}$, as this affects $N^{O L 2}{ }_{I F}$. To evaluate $L_{G B}$, we conducted experiments to measure $T S_{a c c}$. Using prototype systems, we found that the required $L_{G B}$ were about $60 \mathrm{~ns}$ caused by the fluctuation of TS control timing to achieve $\mathrm{TS}_{\text {Sync }}$ for the whole network. On the basis of the above experimental results, we conducted a simulation to evaluate $N^{O L 2}{ }_{I F}$ when changing $N_{M}, N_{L}$ and $T R$ in comparison with $N^{P-O A D M}{ }_{I F}$. For the assumed network model, we found that the OL2SW-NW could reduce the $N_{I F}$ by $30 \%$ at maximum. In view of the above results, we could find that the OL2SW-NW could reduce $N_{I F}$. Therefore, we concluded that the OL2SW-NW could effectively aggregate the traffic while suppressing the CAPEX of future aggregation networks.

\section{References}

[1] Future Internet Public-Private Partnership (FI-PPP), [Online]. Available: https://www.fi-ppp.eu Accessed Dec. 2016.

[2] Future Internet Architectures - Next Phase (FIA-NP), [Online]. Available: https://www.nsf.gov/funding/pgm_summ.jsp?pims_id= 504882 Accessed Dec. 2016.

[3] US Ignite, [Online]. Available: https://www.us-ignite.org/ Dec. 2016.

[4] K. Kitayama, "The Vision Towards Smart Photonic Cloud," Proc. Photonics in Switching (PS), We.III.1-1, Sept. 2015.

[5] G. Zhang, M. De Leenheer, A. Morea, and B. Mukherjee, "A survey on OFDM-based elastic core optical networking," IEEE Commun. Surveys Tut., vol.15, no.1, pp.65-87, 2013.

[6] ITU-T Recommendation G983.1. Broadband optical access system based on Passive Optical Access, Jan. 2005.

[7] IEEE, "802.3av 10Gb/s Ethernet Passive Optical Network (10GEPON)," Oct. 2009.

[8] IEEE 802.3 Ethernet Working Group, "Industry connections feasibility assessment for the next generation of EPON (NG-EPON)," 2015.

[9] M. Ruffini, "Metro-Access Network Convergence," Proc. OFC, Th4B.1, March 2016.

[10] R. Bonk, H. Schmuck, W. Poehlmann, and T. Pfeiffer, "Beneficial OLT transmitter and receiver concepts for NG-PON2 using semiconductor optical amplifiers [invited]," IEEE/OSA J. Opt. Commun. Networking, vol.7, no.3, pp.A467-A473, 2015.

[11] D. Murayama, N. Oota, K.-I. Suzuki, and N. Yoshimoto, "Low latency dynamic bandwidth allocation for $100 \mathrm{~km}$ long reach 10G-EPON," Proc. IEEE Communication Quality and Reliablitation (CQR) Int. Workshop, San Diego, CA, May 2012.

[12] K. Hattori, M. Nakagawa, N. Kimishima, M. Katayama, A. Misawa, and A. Hiramatsu, "Optical Layer-2 switch network based on WDM/TDM nano-sec wavelength switching," Proc. ECOC, We.3.D.5, Sept. 2012.

[13] K. Hattori, M. Nakagawa, N. Kimishima, M. Katayama, and A. Misawa, "Dynamic path bandwidth allocation for $1000 \times 10$-scale optical layer-2 switch network based on hierarchical timeslot allocation algorithm and timeslot converter," Proc. ECOC, Th.2.E.2, Sept. 2013.

[14] K. Hattori, M. Nakagawa, M. Katayama, and H. Ogawa, "Method for synchronizing timeslot of WDM/TDM multi-ring network independent of fiber delay," Proc. OECC, TU4A-5, July 2014.

[15] M. Nakagawa, K. Hattori, N. Kimishima, M. Katayama, and A. Misawa, "Hierarchical Time-Slot Allocation for Dynamic Bandwidth Control in Optical Layer-2 Switch Network," IEICE Trans. Commun., vol.E97-B, no.7, pp.1303-1312, July 2014.

[16] K. Hattori, M. Nakagawa, H. Date, M. Katayama, and H. Ogawa, "Flow Burst Conversion for Large-scale Optical Layer-2 Switch Network based on Scale-out Flow Control Method," Proc. ECOC, P.6.2, Sept. 2014.

[17] K. Hattori, M. Nakagawa, T. Matsuda, M. Katayama, and K. Koda, "Optical TDM Fast Reroute Method for Bufferless Metro Ring Networks with Arbitrary Fiber Length,” Proc. ECOC, P.6.1, Sept. 2015.

[18] K. Hattori, T. Homemoto, M. Nakagawa, N. Kimishima, M. Katayama, and A. Misawa, "Optical Layer 2 Switch Network with Bufferless Optical TDM and Dynamic Bandwidth Allocation," IEICE Trans. Electron., vol.E99-C, no.2, pp.189-202, Feb. 2016.

[19] A. Boskovic, M. Sharma, N. Antoniades, and M. Lee, "Broadcast and select OADM nodes application and performance trade-offs," Proc. OFC, TuX2, 2002.

[20] ITU-T Rec. G.709, "Network Node Interface for the Optical Transport Network (OTN)," 2001. 
[21] D. Chiaroni, R. Urata, J. Gripp, J.E. Simsarian, G. Austin, S. Etienne, T. Segawa, Y. Pointutier, C. Simonneau, Y. Suzaki, T. Nakahara, M. Thottan, A. Adamiecki, D. Neilson, J.C. Antona, S. Bigo, R. Takahashi, and V. Radoaca, "Demonstration of the Interconnection of Two Optical Packet Rings with a Hybrid Optoelectronic Packet Router," Proc. ECOC, PD3.5, Torino, Italy, Sept. 2010.

[22] B. Ušćumlić, Y. Pointurier, A. Morea, and S. Bigo, "On the Cost of Protection in Optical Slot Switching Rings with Elastic Transponders," Proc. OFC, Th2A.46, March 2015.

[23] A. Chen, A.K.-S. Wong, and C.-T. Lea, "On the space reuse efficiency of TSI-free OTDM rings," IEEE Commun. Lett., vol.12, no.4, pp.322-324, April 2008.

[24] J.-L. Ferrant, M. Gilson, S. Jobert, M. Mayer, M. Ouellette, L. Montini, S. Rodrigues, and S. Ruffini, "Synchronous Ethernet: a method to transport synchronization," IEEE Commun. Mag., vol.46, no.9, pp.126-134, Sept. 2008.

[25] C.-J. Chae and T. Jayasinghe, "A Method to Use Continuous Mode Receivers as Burst Mode Receivers for TDM-based Passive Optical Networks," Proc. ECOC, p.129, Sept. 2007.

[26] J. Yang, X. Liu, F. Chu, C. Wei, and J. Gao, "Analysis of an optical packet switch with limited wavelength converters and shared buffers," Proc. ICCSNT, pp.2512-2515, Dec. 2011.

[27] O. Ishida and H. Takahashi, "Loss-imbalance equalization in arrayed-waveguide-grating (AWG) multiplexer cascades," J. Lightw. Technol., vol.13, no.6, pp.1155-1163, June 1995.

[28] K. Taguchi, H. Nakamura, K. Asaka, S. Nakano, S. Kimura, and N. Yoshimoto, "100-ns selective burst-mode transceiver for 40 $\mathrm{km}$ reach symmetric 40-Gbit/s WDM/TDM-PON," ECOC'2013, Mo.4.F.5, 2013.

[29] J. Seoane, A. Siahlo, A. Clausen., K.L. Oxenløwe, and P. Jeppesen, "Ultra high-speed demultiplexing using a NOLM based on commercially available highly non-linear fibre," Proc. ECOC, We.1.5.4, Sept. 2004.

[30] ITU-T G.8032, "Ethernet ring protection switching," 2008.

[31] Agiltron, [Online]. Available: www.agiltron.com/Nanospeed_series \%20F.htm, Accessed Dec. 2016.

[32] K. Okamoto and A. Hiramatsu, "Proposal of a Sub- $\lambda$ Switching Network and its Time-slot Assignment Algorithm for Network with Asynchronous Time-slot Phase," Proc. ITU Kaleidoscope, S7.3, April 2013.

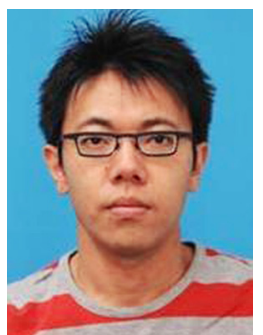

Kyota Hattori received his B.E. in applied physics and the M.E. in computational science and engineering from Nagoya University, Aichi, Japan, in 2004 and 2006, respectively. In 2006, he joined NTT Corporation, Tokyo, Japan. He is currently with NTT Network Service Systems Laboratories, where he is engaged in research and development on a transport network system. He received the Award for SC4 Best Paper from Photonics in Switching 2012 (PS2012) and the Young Researcher's Award from IEICE in 2013.

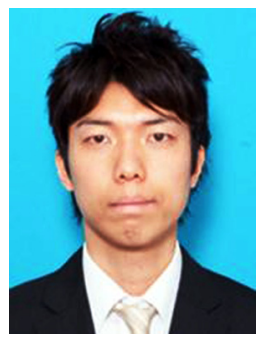

Masahiro Nakagawa received his B.E. and M.E. in electrical engineering and computer science from Nagoya University, Aichi, Japan, in 2008 and 2010, respectively. In 2010, he joined NTT Corporation, Tokyo, Japan. Currently, he is with NTT Network Service Systems Laboratories, where he is involved in research and development on a transport network system. His research interests include photonic network systems and dynamic network control. He is a member of IEICE.

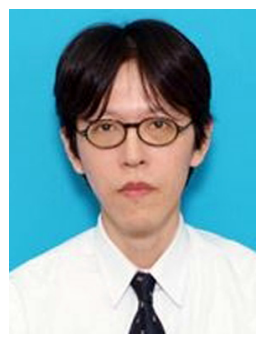

Toshiya Matsuda received his B.E. and M.E. in electrical information and communication engineering from Waseda University in 1990 and 1992, respectively. In 1992, he joined NTT Transmission Systems Laboratories and engaged in research on long-haul large-capacity transmission systems. He is currently a senior research engineer in NTT Network Service Systems Laboratories.

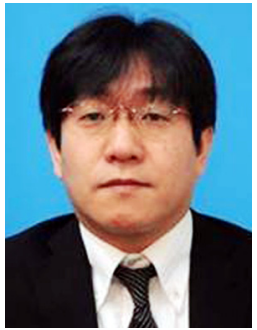

Masaru Katayama received his B.E. and M.E. degrees from Hokkaido University in 1990 and 1992, respectively. He joined NTT Corporation, Tokyo, Japan, in 1992 and is currently a senior research engineer and supervisor at NTT Network Service Systems Laboratories. He has been researching high-performance IP packet processing systems with field programmable hardware and photonic network systems. His other current research interests are a photonic layer 2 switching system based on TDM and its control system. He is a member of IEICE and IEEE.

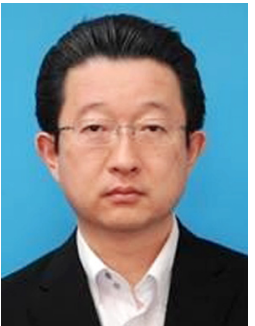

Katsutoshi Koda received the B.S. and M.S. in mechanical sciences from the Tokyo University of Science in 1987 and 1989, respectively. After joining the Nippon Telegraph and Telephone Corporation (NTT) in 1989, he was engaged in the development of transport systems at the Network System Development Center and Network Service Systems Laboratories. He has developed transport network systems. Currently, he is engaged in research and development for future optical networking and transport network management technology. 